\title{
A Janus-Faced Bacterium: Host-Beneficial and -Detrimental Roles of Cutibacterium acnes
}

\begin{abstract}
Holger Brüggemann ${ }^{1 *}$, Llanos Salar-Vidal ${ }^{2}$, Harald P. M. Gollnick ${ }^{3}$ and Rolf Lood ${ }^{4 *}$
${ }^{1}$ Department of Biomedicine, Aarhus University, Aarhus, Denmark, ${ }^{2}$ Department of Clinical Microbiology, Fundacion Jimenez Diaz University Hospital, Madrid, Spain, ${ }^{3}$ Department of Dermatology and Venerology, Otto-von-Guericke University Magdeburg, Magdeburg, Germany, ${ }^{4}$ Division of Infection Medicine, Department of Clinical Sciences, Lund University, Lund, Sweden
\end{abstract}

The bacterial species Cutibacterium acnes (formerly known as Propionibacterium acnes) is tightly associated with humans. It is the dominant bacterium in sebaceous regions of the human skin, where it preferentially colonizes the pilosebaceous unit. Multiple strains of $C$. acnes that belong to phylogenetically distinct types can co-exist. In this review we summarize and discuss the current knowledge of $C$. acnes regarding bacterial properties and traits that allow host colonization and play major roles in host-bacterium interactions and also regarding the host responses that $C$. acnes can trigger. These responses can have beneficial or detrimental consequences for the host. In the first part of the review, we highlight and critically review disease associations of C. acnes, in particular acne vulgaris, implant-associated infections and native infections. Here, we also analyse the current evidence for a direct or indirect role of a $C$. acnes-related dysbiosis in disease development or progression, i.e., reduced C. acnes strain diversity and/or the predominance of a certain phylotype. In the second part of the review, we highlight historical and recent findings demonstrating beneficial aspects of colonization by $C$. acnes such as colonization resistance, immune system interactions, and oxidant protection, and discuss the molecular mechanisms behind these effects. This new insight led to efforts in skin microbiota manipulation, such as the use of $C$. acnes strains as probiotic options to treat skin disorders.

Keywords: Cutibacterium acnes, acne (acne vulgaris), implant-associated infection, skin microbiome, beneficial bacteria, Propionibacterium acnes

\section{INTRODUCTION}

Cutibacterium acnes (C. acnes) is a Gram-positive member of the skin microbiota and as such, a very prevalent microorganism associated with humans. It is a lipophilic microorganism, and the most dominant bacterium in sebaceous, lipid-rich areas of normal human skin; in addition, it is also very abundant on moist and dry skin areas (Byrd et al., 2018). It is also found in several other organs and tissue sites, such as oral cavity, stomach, lung, urinary tract, and prostate (Sasaki et al., 1980; Shannon et al., 2006; Delgado et al., 2011; Davidsson et al., 2016); however, it is unclear if the organism can live and thrive in sites other than the skin or if its detection is a result from skin-derived carry-over, sample contamination, or a temporary breach.

The colonization of $C$. acnes, or rather the fact that human defense systems allow $C$. acnes to colonize the largest organ, suggests that the bacterium does not harm the human host, at least not 
under normal circumstances. C. acnes grows particular well in sebaceous rich areas; during the development of puberty, with increased sebaceous gland activity, C. acnes predominantly colonizes those skin areas with preference of infrainfundibula of sebaceous follicles (Leyden et al., 1998). The successful colonization by $C$. acnes could indicate that the bacterium actually has host-beneficial roles. In this review, we highlight some of the known and suggested beneficial functions of $C$. acnes. On the other hand, most, if not all, bacteria can cause harm to the human host under certain conditions such as in a predisposed or immunocompromised state. This also holds true for $C$. acnes, in particular if bacteria breach the skin surface and reach deeper tissue sites. However, we still know very little about the active roles that $C$. acnes might have in disease formation or progression; even for the skin condition acne vulgaris (AV) there are still open questions regarding the exact involvement of $C$. acnes on the molecular level. This review aims at a description and evaluation of some disease associations of $C$. acnes (Figure 1, Table 1).

In the last decades, improvement of technologies such as next generation sequencing (NGS) has provided new possibilities to study C. acnes and the skin microbiome. Full genome sequencing of many different strains has highlighted the pan-genome and the genetic repertoire of $C$. acnes (Tomida et al., 2013; Scholz et al., 2016). This has led to the identification of host-interacting, secreted, and/or surface-exposed proteins of C. acnes as well as other molecules and metabolites. This review contains current knowledge of bacterial factors thought to be important in hostbeneficial or -detrimental functions. In addition, sequencing of almost 300 genomes (status February 2021) has provided a deep insight into the population structure of this species. Results of the phylogenomic analysis and the comparison with genomes of other propionibacteria, in particular the classical diary propionibacteria, e.g., Propionibacterium freudenreichii, have led to a proposal of changing the species name from Propionibacterium acnes to Cutibacterium acnes (Scholz and Kilian, 2016). This name change provoked a controversy among scientists; among others, it was noted that a new species name might generate some confusion among clinicians (Alexeyev et al., 2018).

Among $C$. acnes, we distinguish six main phylotypes, often labeled as $\mathrm{IA}_{1}, \mathrm{IA}_{2}$, IB, IC, II, and III (McLaughlin et al., 2019). Several typing schemes to distinguish strains of $C$. acnes exist, traditionally based on multi-locus sequence typing (MLST) schemes and now more and more replaced by whole genomebased phylotyping (Lomholt and Kilian, 2010; McDowell et al., 2011; Scholz et al., 2014). Three subspecies are currently distinguished, subspecies acnes (comprising phylotypes $\mathrm{IA}_{1}, \mathrm{IA}_{2}$, IB, IC), subspecies defendens (phylotype II) and subspecies elongatum (phylotype III) (Dekio et al., 2019). Based on the core genome phylogeny of $C$. acnes, a single-locus sequence typing (SLST) scheme was created that can differentiate 10 lineages in total (types A-L); the complex phylotype $\mathrm{IA}_{1}$ is further split into five SLST types (A-E) (Scholz et al., 2014). The other SLST types correspond to the phylotypes as follows: F, $\mathrm{IA}_{2}$; G, IC; H, IB; K, II; L, III (Figure 2). This review will often refer to the main six phylotypes (and the 10 SLST types) and their distinct associations with health and disease. Apart from core genome differences, the accessory genome of $C$. acnes is relatively small but comprises elements such as a linear plasmid (designated p15.1.R1 or pIMPLE-HL096PA1, encoding a conjugation apparatus and a tight adherence pili locus) and a circular plasmid (designated pTZC1, encoding genes conferring resistance to macrolides, clindamycin and tetracycline) (Tomida et al., 2013; Davidsson et al., 2017; Aoki et al., 2020). In addition, around 60 other regions that are not part of the core genome can be identified in the pan-genome of $C$. acnes; these non-core genes are often, but not exclusively, phylotype-specific, and code for a variety of functions such as different transport systems, bacteriocin synthesis, resistance to heavy metals and antibiotics, restriction modification systems, CRISPR/cas systems, (cryptic) prophages, transposases, carbohydrate and amino acid processing enzymes, and many so far unknown functions (Brüggemann et al., 2012; Tomida et al., 2013; Scholz et al., 2016).

\section{MR. HYDE-THE PATHOGENIC SIDE OF C. acnes: PROVEN AND SUGGESTED DISEASE ASSOCIATIONS AND MECHANISMS}

\section{General Considerations}

Hundreds of studies have been carried out to shed light on the potential role of $C$. acnes in various diseases. Due to the ubiquitous bacterium's presence on human skin (Byrd et al., 2018), many efforts focused on skin disorders, in particular AV. Mainly in the last two decades several non-skin diseases were reported to be associated with $C$. acnes, including implantassociated infections (IAIs), primary joint and bone infections, sarcoidosis, and prostate cancer (Figure 1).

Here, we do not aim at the presentation of the entire literature that reported evidence and counter-evidence for a role of $C$. acnes in disease formation and/or progression; rather, this review will present and discuss selected disease associations of $C$. acnes. The selection is based on the availability of recent advances, as well as new findings that shed light on $C$. acnes' role and its host-interacting properties.

\section{Skin Disorders Associated With C. acnes C. acnes and Acne Vulgaris}

Historically, much effort has been undertaken to pinpoint the exact role of $C$. acnes in the onset and/or progression of AV. Many hypotheses and possible mechanisms have been suggested, but solid evidence is still scarce. On the other hand, there is no case of AV without $C$. acnes. There is strong evidence that therapeutically induced reduction of $C$. acnes' population (e.g., directly by antibiotics or indirectly by retinoids that suppress sebum production) diminish inflammation. The reader is referred to a few recent reviews that summarize diverse aspects on the debated role of C. acnes in AV (Dréno et al., 2018, 2020; Platsidaki and Dessinioti, 2018; Brüggemann, 2019; McLaughlin et al., 2019).

Here, a few selected features regarding C. acnes' association with AV should be highlighted. In the last decade, the concept 

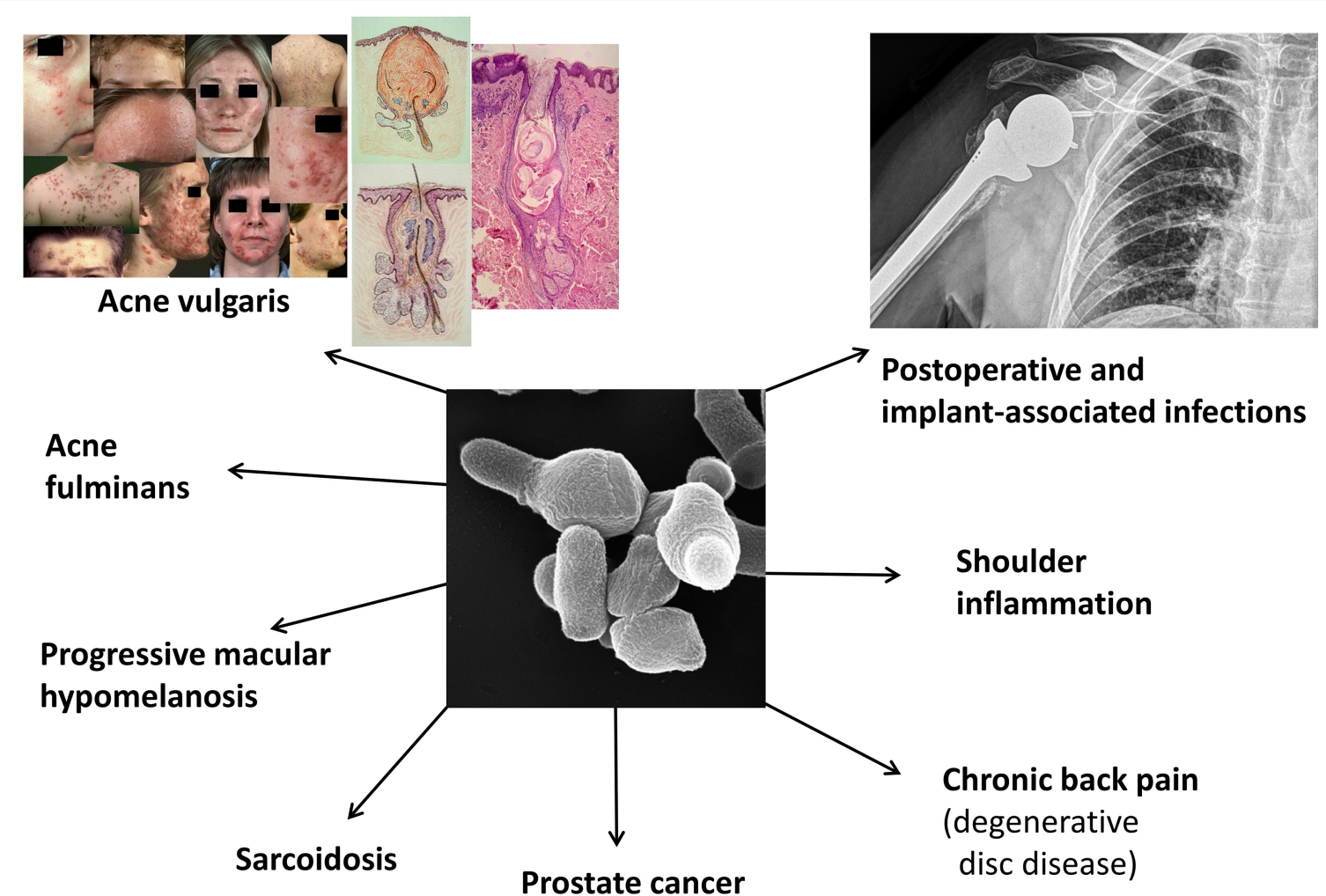

FIGURE 1 | Known or suspected disease associations of C. acnes. Shown are currently investigated and debated disease associations of $C$. acnes. In this review we mainly focus on acne vulgaris, implant-associated infections, and a few native infections. The clinical pictures regarding acne vulgaris are taken from Gollnick and Zouboulis (2014).

of a dysbiosis of the C. acnes population on acne-affected skin compared to normal skin was put forward; such a shift of the $C$. acnes population was -to some extent- observed with culture-dependent and culture-independent methods (Lomholt and Kilian, 2010; McDowell et al., 2011; Fitz-Gibbon et al., 2013; Dréno et al., 2020). In these studies, it was stated that strains belonging to certain phylotypes of $C$. acnes are $\mathrm{AV}$ associated, while other strains belonging to different phylotypes are preferentially associated with healthy skin. More accurately, in case of $\mathrm{AV}$-affected skin compared to normal skin, a higher relative abundance of certain strains, in particular strains of phylotype $\mathrm{IA}_{1}$ (and phylotype $\mathrm{IA}_{2}$ ) was observed. However, strains of phylotype $\mathrm{IA}_{1}$ are, in average, also more abundant on healthy (facial) skin than strains belonging to the other phylogenetic lineages, i.e., phylotypes $\mathrm{IA}_{2}$, IB, IC, II, and III (McLaughlin et al., 2019). So far, not many studies have used the SLST typing scheme to determine the phylogeny of acneassociated strains. A recent study has reported the association of the SLST type A (a subset of $\mathrm{IA}_{1}$ strains, Figure 2) with acne-affected skin; however, this study only processed a rather small cohort $(n=36)$ (Dagnelie et al., 2018). In contrast, studies with Japanese patients have detected the SLST type F $\left(\mathrm{IA}_{2}\right)$ as strongly acne-associated (Nakase et al., 2017, 2020), suggesting geographic differences between Europe and Asia. In addition, the SLST type $\mathrm{C}\left(\mathrm{IA}_{1}\right)$ is enriched among acne patients; this SLST type corresponds to the (MLST-based) phylogenetic lineage CC3 (Lomholt and Kilian, 2010; McLaughlin et al., 2019).

It is fairly unlikely that phylotype $\mathrm{IA}_{1}$ strains (or rather strains of SLST types $\mathrm{A}$ and $\mathrm{C}$ ) or phylotype $\mathrm{IA}_{2}$ strains (SLST type F) isolated from acne-associated skin have per se different properties than phylotype $\mathrm{IA}_{1} / \mathrm{IA}_{2}$ strains isolated from healthy skin. In line with this, a study found no differences in the (core as well as accessory) genomes of phylotype $\mathrm{IA}_{1}$ strains isolated from acne and healthy skin, respectively (Lomholt et al., 2017). Moreover, there is no solid evidence that supports the assumption that phylotype $\mathrm{IA}_{1} / \mathrm{IA}_{2}$ strains are per se more virulent compared to strains of other phylotypes, even though such differences on the strain level might exist. Studies that have compared strain properties of C. acnes (comparing either strains belonging to the same phylotype or strains belonging to different phylotypes) regarding their pathogenic potential came to conflicting results (Nagy et al., 2005; Jasson et al., 2013; Lheure et al., 2016; Agak et al., 2018). Current studies that report strain comparisons need to be handled with caution; much can depend on the individual, selected strains (that can have slight differences, e.g., in growth behavior and oxygen sensitivity) as well as on the method/model that is applied to evaluate bacterial pathogenicity or host-interaction. 
TABLE 1 | Disease associations of $C$. acnes and evaluation of the existing evidence.

\begin{tabular}{|c|c|c|c|c|}
\hline Disease & Level of evidence* & $\begin{array}{l}\text { Phylotype association } \\
\text { (enrichment) }\end{array}$ & Selected reviews & $\begin{array}{l}\text { Selected primary } \\
\text { literature }\end{array}$ \\
\hline Acne vulgaris & $\begin{array}{l}\text { A } \\
\text { Disease association } \\
\text { Detection }(\mathrm{CD}, \mathrm{Cl}, \mathrm{HC} / \mathrm{F})^{\star \star} \\
\text { Skin model } \\
\text { Animal model (with } \\
\text { limitations) } \\
\text { Responsive to } \\
\text { antibiotic treatment }\end{array}$ & $\begin{array}{l}\text { IA } A_{1} \text { (SLST types A andC; } \\
\text { possibly also SLST types D } \\
\text { and E) and, possibly, IC } \\
\text { (SLST type G) }\end{array}$ & $\begin{array}{l}\text { Dréno et al., 2018, 2020; } \\
\text { Platsidaki and Dessinioti, } \\
\text { 2018; Brüggemann, 2019; } \\
\text { McLaughlin et al., } 2019\end{array}$ & $\begin{array}{l}\text { Lomholt and Kilian, } \\
\text { 2010; McDowell et al., } \\
\text { 2011; Fitz-Gibbon } \\
\text { et al., 2013; Dagnelie } \\
\text { et al., 2018 }\end{array}$ \\
\hline $\begin{array}{l}\text { Progressive macular } \\
\text { hypomelanosis }\end{array}$ & $\begin{array}{l}\text { B } \\
\text { Disease association } \\
\text { Detection }(\mathrm{CD} \text { and } \mathrm{Cl})^{\star \star} \\
\text { Responsive to } \\
\text { antibiotic treatment }\end{array}$ & III (SLST type L) & McDowell et al., 2020 & $\begin{array}{l}\text { Barnard et al., 2016; } \\
\text { Petersen et al., } 2017\end{array}$ \\
\hline $\begin{array}{l}\text { Spine instrumentation } \\
\text { infections } \\
\text { Spine osteomyelitis }\end{array}$ & $\begin{array}{l}\text { B } \\
\text { Disease association } \\
\text { Detection }(C D)^{\star \star} \\
\text { Responsive to antibiotic } \\
\text { treatment (with limitations) }\end{array}$ & $\begin{array}{l}\mathrm{IA}_{1} \text { (SLST type A) } \\
\mathrm{IA}_{2}(\mathrm{SLST} \text { type } \mathrm{F})\end{array}$ & Khalil et al., 2019 & Uçkay et al., 2010 \\
\hline $\begin{array}{l}\text { Disc degeneration and } \\
\text { Modic type } 1 \text { changes }\end{array}$ & $\begin{array}{l}\text { B } \\
\text { Disease association } \\
\text { Detection }(\mathrm{CD}, \mathrm{Cl}, \mathrm{HC} / \mathrm{IF})^{\star \star} \\
\text { Animal model } \\
\text { Responsive to antibiotic } \\
\text { treatment (with limitations) }\end{array}$ & $\begin{array}{l}\left.\mathrm{IA}_{1} \text { (SLST type } \mathrm{A}\right) \\
\text { ॥ (SLST type } \mathrm{K})\end{array}$ & $\begin{array}{l}\text { Capoor et al., 2019; } \\
\text { Manniche and O'Neill, 2019; } \\
\text { Jha and Sairyo, } 2020\end{array}$ & $\begin{array}{l}\text { Albert et al., 2013; } \\
\text { Capoor et al., 2017; Lin } \\
\text { et al., 2018; } \\
\text { Ohrt-Nissen et al., } \\
2018\end{array}$ \\
\hline
\end{tabular}

${ }^{*} A, \quad B, \quad C:$ strong, medium, and weak evidence, respectively, based on the existing scientific literature. ${ }^{* *} \mathrm{CD}$, culture-dependent; Cl, culture-independent; IHC/IF, immunohistochemistry/immunofluorescence.

The relative enrichment of phylotype $\mathrm{IA}_{1} / \mathrm{IA}_{2}$ strains in acne could also have another explanation: the reduction of strains belonging to other phylotypes. An overall reduction of C. acnes strain diversity could be associated with $\mathrm{AV}$; this implies that not one or a few $C$. acnes phylotypes are disease-associated but rather the lack of strain/phylotype diversity. However, a clear-cut, solid large scale study with 100's of early-stage acne patients and matched healthy controls is lacking; such a study should also take possible confounders into consideration, such as past and current treatments, since most acne suffers use some sort of (topical) treatment. A recent study indicated that loss of strain/phylotype diversity might go along with increased innate immune stimulation: a three-strain mixture (strains of phylotypes $\mathrm{IA}_{1}$, II, and III) elicited a weaker innate immune response in healthy skin explants than the three strains applied individually (Dagnelie et al., 2019). Regarding bacterial properties and factors that could influence the formation and/or progression of $\mathrm{AV}$, some of them will be presented below, including CAMP factors, biofilm formation, porphyrin, and short-chain fatty acid production. Figure 3 summarizes a tentative model of the role of C. acnes in AV.

Taken together, the concept of an acne-associated $C$. acnes dysbiosis on the phylotype/strain level leading to a diversity loss seems likely but needs further proof. If the 


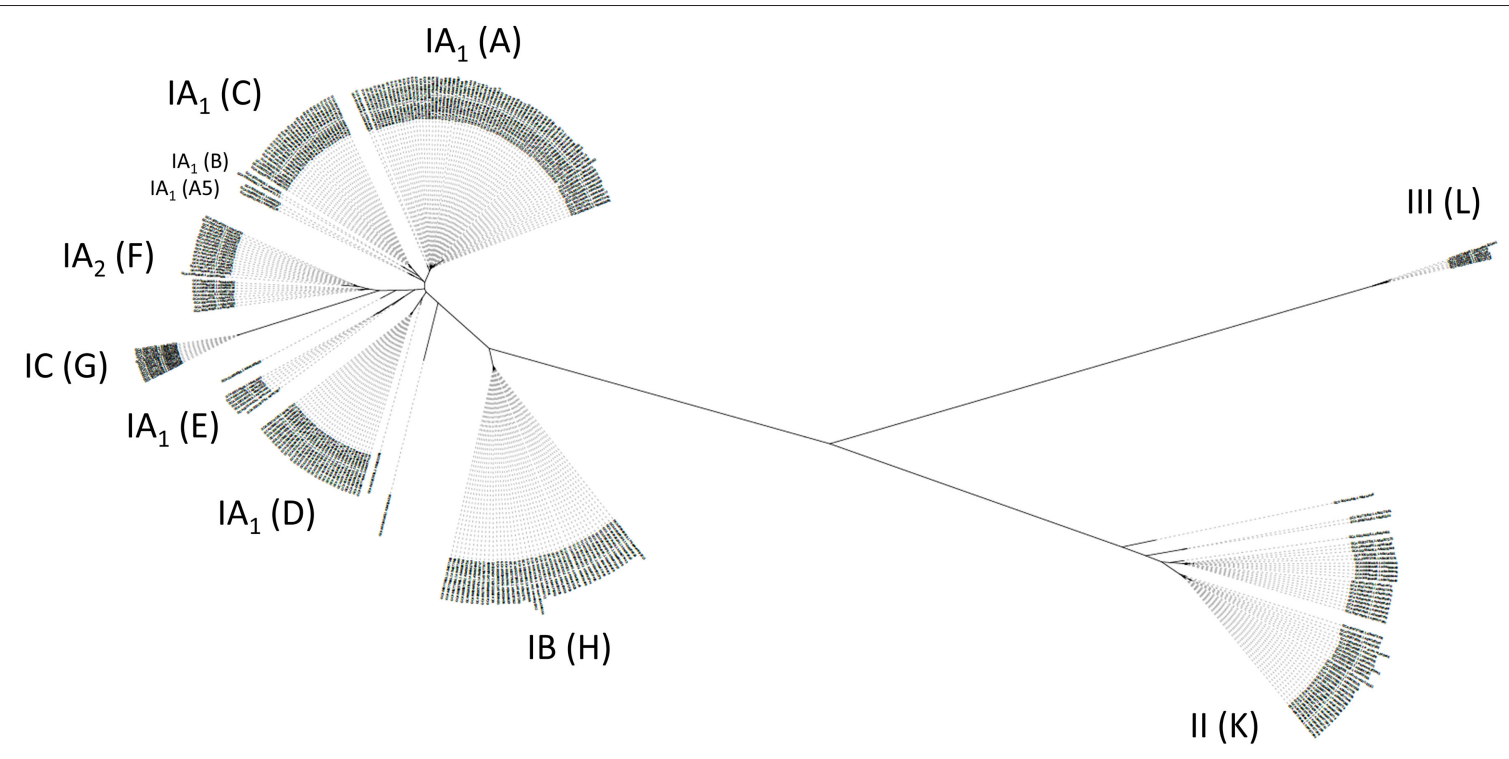

FIGURE 2 | Diversity of the population of $C$. acnes. A population tree is shown based on a phylogenomic comparison relying on core genome-located nucleotide polymorphisms. Distinct phylotypes of $C$. acnes are highlighted as six main phylotypes $I A_{1}, I A_{2}$, IB, IC, II, III, and 10 SLST types A-L. SLST types F, G, H, K, L correspond to phylotypes IA $\mathrm{A}_{2}, \mathrm{IC}$, IB, II, and III, respectively. Please note that SLST types A-E are distinct clades of phylotype IA $A_{1}$. In particular, SLST types D and E are distinct from acne-associated SLST types A-C. In total, 286 publicly available C. acnes genomes were analyzed and the closed genome of strain KPA171202 (phylotype IB, SLST type H) was taken as reference [modified from Brüggemann (2019)].

reduction of phylotype/strain diversity in acne-affected skin holds true, as current data suggests, it would imply that a diverse $C$. acnes population is a marker of healthy skin and such diversity thus may be beneficial for skin heath. The question then remains how a high strain diversity could contribute to health, e.g., which mechanisms are at play. Since different phylotypes of $C$. acnes are reported to have different microbiological, and -to some extentdifferent host-interacting properties (Nagy et al., 2005; Jasson et al., 2013; Lheure et al., 2016; Agak et al., 2018; Dekio et al., 2019), it can be speculated that a diverse C. acnes population can compensate or dampen phylotype-specific peculiarities, metabolize different components, provide complementary beneficial traits and/or undergo some sort of symbiotic relationship.

\section{Other Skin-Disease Associated With C. acnes}

C. acnes is suspected to be involved in other skin diseases. The disease progressive macular hypolmelanosis (PMH) is characterized by non-scaly hypopigmented skin areas that are visible in the sebaceous areas; often the lower back skin is affected. Regarding the role of $C$. acnes in PMH the reader is referred to a recent review (McDowell et al., 2020) that, among others, summarized and discussed data from primary studies (Barnard et al., 2016; Petersen et al., 2017). In brief, a rather clear enrichment of certain strains of $C$. acnes was seen in PMH lesions; these strains belong to phylotype III (SLST type L) of $C$. acnes. This phylotype (renamed to C. acnes subsp. elongatum) is poorly investigated so far; it is quite different from the other main phylotypes, also regarding its morphology (Dekio et al., 2019).
C. acnes might also play a role in acne fulminans, a rare, severe form of inflammatory acne, associated with painful ulceration, and in some cases with systemic signs. A recent study identified the SLST type A (phylotype $\mathrm{IA}_{1}$ ) in about $60 \%$ of patients (Bocquet-Trémoureux et al., 2020); however, as discussed above, the SLST type A is often found to be the dominating type in healthy skin as well. Thus, at present, there is no strong evidence that (a specific phylotype of) C. acnes is a driver of disease.

\section{Non-skin Diseases Associated With C. acnes \\ C. acnes and Implant-Associated Infections}

More and more studies report the detection of C. acnes in IAIs. A few reviews have summarized the current knowledge regarding the role of C. acnes in IAIs (Portillo et al., 2013; Achermann et al., 2014; Aubin et al., 2014; Gharamti and Kanafani, 2017; Boisrenoult, 2018; Renz et al., 2018; Lin et al., 2020). It seems that $C$. acnes is now more often found in IAI-associated specimens than two decades ago. The reason for this increase is most likely related to changed procedures and diagnostic tools. One important change is the introduction of sonication of removed periprosthetic tissue specimens or removed medical devices before microbial cultivation (Trampuz et al., 2007). This technique is nowadays more often used in everyday clinical microbiology practice in many hospitals; it has been shown to increase the bacterial recovery from specimens (Trampuz et al., 2007; Esteban et al., 2008). In addition, cultivation procedures have been adapted to guarantee also the reliable detection of slow-growing anaerobic bacteria such as $C$. acnes; e.g., cultivation times have often been extended to 14 and even 21 days (Bossard et al., 2016; Kvich et al., 2016). Moreover, due to 
the introduction of MALDI-TOF mass spectrometry for bacterial species determination in the last decade, the identification of cultivated bacteria has become more easily, and samples with polymicrobial growth can be analyzed more comprehensively. Furthermore, the use of multiplex PCR techniques has also aided to improve the detection of low-virulent microorganisms such as C. acnes (Morgenstern et al., 2018; Sigmund et al., 2019).

\section{Prosthetic Joint Infections}

C. acnes is associated with around $10 \%$ of all prosthetic joint infections (PJIs), being more frequently isolated in late-chronic infections (Benito et al., 2019; Triffault-Fillit et al., 2019). The shoulder is the most frequent site of isolation, probably due to the greater rate of colonization in the axillary region than in the hip or knee (Boisrenoult, 2018; Lin et al., 2020). It is now estimated that $C$. acnes accounts for $31-70 \%$ of all PJIs after shoulder arthroplasty (Fink and Sevelda, 2017). C. acnes is also believed to be one of the main opportunistic pathogens involved in latent postoperative infections in spinal instrumentation surgeries (Khalil et al., 2019).

The diagnosis of C. acnes-associated PJI is difficult, mainly due to the lack of specific local inflammatory signs at the surgical site of infection. Persistent unexplained pain is often the only symptom; evidence of implant loosening is seen in a great number of cases (Shah et al., 2015; Lin et al., 2020). Even though $C$. acnes is primarily associated with low-grade, chronic or delayed infections, it can also cause acute infections (Zeller et al., 2007; Dodson et al., 2010). Systemic symptoms, such as fever are not frequent and inflammatory markers are normal or slightly elevated. Only half of the patients presented local signs of infections (Zeller et al., 2007; Lavergne et al., 2017). This might explain why C. acnes can be unexpectedly found in revision surgery (Benito et al., 2019). Clinical signs of infection are more often encountered during the first two years after arthroplasty procedure (Zeller et al., 2007). Nodzo et al. reported that shoulder PJIs present with a less robust host peripheral inflammatory response in comparison with knee and hip PJIs (Nodzo et al., 2017). Rates of infections are higher in males (Figa et al., 2017; Renz et al., 2018). One reason might be related to gender-specific differences regarding the distribution and frequency of hair follicles (Hudek et al., 2016). Abnormal radiographic findings are usually observed in a minority of cases of shoulder PJIs (Piggott et al., 2015; Shields et al., 2016). It is still unclear if humeral loosening may indicate a $C$. acnes infection. Radiographic findings (e.g., in C. acnes-associated spinal infections) may show the formation of a "halo" around screws, osteolysis or evidence of pseudoarthrosis of the fusion mass (Khalil et al., 2019).

\section{Cardiac Device-Related Infections}

C. acnes has also been described as a causative agent of cardiovascular device-related infections, involving prosthetic heart valves, permanent pacemakers, prosthetic valve rings, or implantable cardioverter-defibrillators. Diagnosis of C. acnesassociated infective endocarditis (IE) is complicated, due to the indolent nature of the infection, the slow growth rate of the microorganism and its consideration as a common contaminant in blood cultures (Clayton et al., 2006). In the C. acnes-associated IE cohort of Banzon et al. invasive disease was reported in $78 \%$ of the cases and embolic complications in 36\% (Banzon et al., 2017), whereas in the study of Sohail et al. myocardial abscesses occurred in $36 \%$ of the cases (Sohail et al., 2009). Extended incubation of blood cultures is recommended to increase the microbiological yield. Banzon et al. showed that valve sequencing may aid in the identification of $C$. acnes-associated IE, in particular in culture negative cases (Banzon et al., 2017).

\section{Breast Implant Infections}

Capsular contracture is a frequent and severe complication following breast implantation. The etiology is not fully understood, but studies that employed sonication of the implant, have suggested that bacterial colonization is associated with capsular contracture (Reischies et al., 2017). Tamboto et al. demonstrated in a porcine model a relation between subclinical infection, bacterial biofilm formation and capsular contracture (Tamboto et al., 2010). The most common bacteria isolated in these samples are microorganisms from the skin microbiota, being $C$. acnes one of the most recovered ones (del Pozo et al., 2009; Karau et al., 2013). In the study of Lee et al. C. acnes was found as the most prevalent microorganism in cases with chronic infection (Lee et al., 2020).

\section{Neurosurgical Shunt Infections}

C. acnes is considered an emerging opportunistic pathogen in neurosurgery procedures (Nisbet et al., 2007). It may be responsible for $\sim 15 \%$ of infections associated with shunt tubular devices, that drain cerebrospinal fluid (CSF) from cerebral ventricles to other body sites, usually the peritoneum (Conen et al., 2008; Bayston et al., 2010). Clinical symptoms in shunt infections are non-specific; the absence of fever is common (Aubin et al., 2014). PCR has been described as effective method to detect C. acnes in CSF infections (Suzuki et al., 2020a), although this method might be too sensitive.

\section{Molecular Typing of C. acnes Isolates From IAIs}

Sequence types (ST) or clonal complexes (CC) have been determined for a large population of $C$. acnes-associated IAI strains in order to establish an association between certain phylotypes and infection. It has been shown that phylotypes IB and II were more frequently isolated from PJIs (McDowell et al., 2012; Aubin et al., 2017). Regarding the CCs, Aubin et al. described CC36 (phylotype IB, SLST type H) and CC53 (phylotype II, SLST type K) as predominant in their cohort. In contrast, in the study of Littorin et al. CC18 (phylotype IA 1 , more specifically SLST type A) was found to be predominant, followed by CC53 (Liew-Littorin et al., 2019). The study of El Sayed et al. found that CC18 was the most abundant CC, followed by CC36 (el Sayed et al., 2019). In spine instrumentation infections, $C$. acnes strains mostly belonged to phylotypes $\mathrm{IA}_{1}(\mathrm{CC} 18)$ and $\mathrm{IA}_{2}$ (CC28; SLST type F) (Aubin et al., 2017). Taken together, these results are contradictory and might indicate that there is no predominate PJI-specific phylotype, implying that all C. acnes 


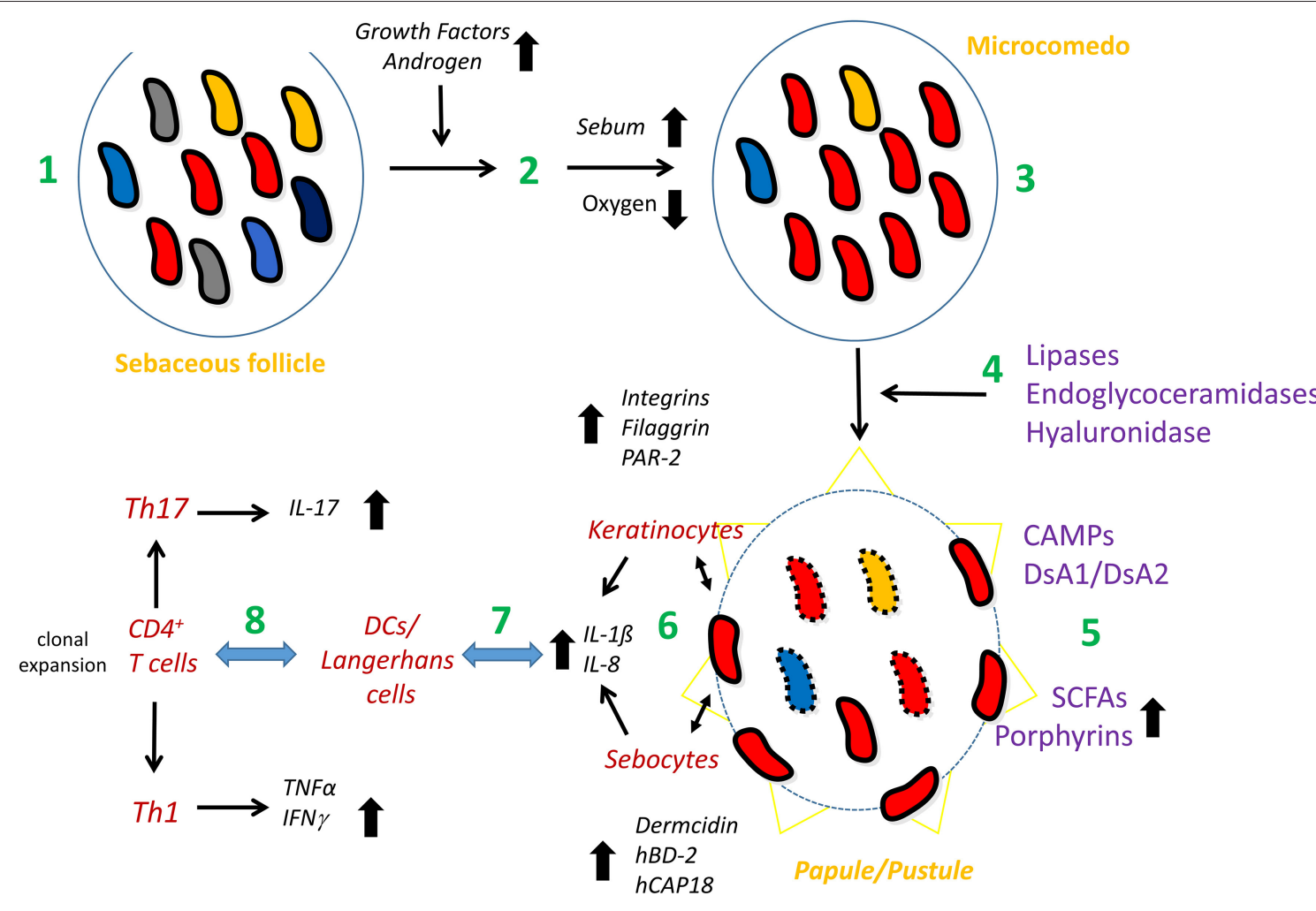

FIGURE 3 | Model of the possible involvement of $C$. acnes in acne vulgaris. The healthy pilosebaceous unit is colonized with a mixture of different $C$. acnes phylotypes/strains (1). Androgen and growth hormone levels in the puberty age rise; these activate sebaceous glands to produce more sebum (2). Exceeding sebum and hyperkeratinization lead to the clogging of the sebaceous duct, the infrainfundibulum, and therefore, to microcomedo formation (acne precursor lesion). The follicular homeostasis is disrupted; the microenvironment of the microcomedo is more anaerobic, providing an advantage and/or disadvantage for different $C$. acnes phylotypes/strains, resulting in the predominance of type IA 1 (SLST types $A$ and C) and/or IA 2 (SLST type F) strains in the comedo (3). Type IA $1 / A_{2}$ strains produce and secrete host-tissue degrading enzymes such as a type IA-specific hyaluronidase, endoglycoceramidases and lipases; the latter lead to the accumulation of free fatty acids (4). In addition, type I $\mathrm{A}_{1} / \mathrm{IA}$ 位 strains produce CAMP factors (CAMP1 and 2) as secreted and cell surface-attached proteins and the adhesive surface glycoproteins dermatan-sulfate adhesins/fibrinogen-binding proteins (DsA1/DsA2). They also secrete short-chain fatty acids such as propionate and produce porphyrins at higher levels (5). These bacterial properties further modulate the follicular microenvironment and pave the way for a closer contact of the bacterium with the cellular microenvironment of the follicle (6), including keratinocytes and, possibly, sebocytes. The direct (bacterial surface) or indirect (secreted factors) contact of $C$. acnes with these cells activates a local innate immune response, mainly in a TLR2-dependent manner, resulting in the release of chemokines/cytokines, such as $\mathrm{IL}-8, \mathrm{IL}-1 \beta$ and others, and can also lead to the production of defense factors (6). Skin-resident DCs/Langerhans cells and macrophages sense and/or are recruited to and infiltrate the irritated tissue site (7). They also interact with skin-resident CD4 ${ }^{+} \mathrm{T}$ cells, resulting in clonal expansion (C. acnes clone-specific proliferation of T-cells). Mixed Th1/Th17 responses result in the secretion of other cytokines including IFN- $\gamma$ and IL-17 (8). Together, this leads to the formation of papules and pustules as seen in inflammatory acne.

types might have the potential to cause PJIs and it rather depends on the individual strain.

\section{C. acnes in IAIs: True Etiology or Sample Contamination?}

C. acnes is considered a low-virulent microorganism that causes infections with subtle clinical presentation, making positive cultures for $C$. acnes in IAIs difficult to interpret. Indeed, the significance of its detection is not always clear because it is considered a common contaminant due to its omnipresence in man-made environments. It has also been reported that $C$. acnes is a possible commensal of the human shoulder joint (Hudek et al., 2020), fueling further the debate whether it represents a contaminant, a passive/transient colonizer or bystander or whether it accounts for true infection. Given the high rates of positive cultures after primary and secondary shoulder arthroplasty, Namdari et al. demonstrated that the most frequently isolated microorganisms from shoulder revision arthroplasty (C. acnes and coagulase-negative staphylococci) are also the most common bacterial contaminants from air in the operating room (Namdari et al., 2020). A careful interpretation of the culture results is needed to distinguish a true-positive from a false-negative result. Parameters, like the number of culturepositive specimens per patient, the cultivation time, and the colony-forming unit (CFU) count help to make this distinction. Frangiamore et al. and Salar-Vidal et al. suggested that clinically relevant isolates of $C$. acnes need a shorter incubation period in comparison with probable contaminants (Frangiamore et al., 2015; Salar-Vidal et al., 2020). On the other hand, a long incubation time could be needed to reactivate dormant cells of $C$. acnes and/or intracellular or biofilm-embedded C. acnes. 
However, not much is currently known about such different states of $C$. acnes.

It is widely accepted that the number of culture-positive specimens per patient is a measure to evaluate the probability of infection (Bémer et al., 2008; Asseray et al., 2010; Frangiamore et al., 2015). In addition, the CFU count may be taken into consideration, as contaminants in most cases often result in very low CFU counts (Esteban et al., 2013; Burnham et al., 2017; Salar-Vidal et al., 2020). In fact, the use of implant sonication fluid alone could increase the rate of false positives if no CFU threshold is established (Grosso et al., 2018). However, neither long cultivation times nor low CFU counts serve as definitive markers of contamination. Each patient should be evaluated individually and interpretations should be done in combination with other relevant clinical and histopathological findings.

\section{Native Infections by $C$. acnes Spine Infections}

C. acnes has been rarely associated with native infections of the spine. Native spine osteomyelitis or spondylodiscitis remains difficult to diagnose. Patients with an infection potentially caused by $C$. acnes usually present with lower back pain without any associated systemic symptoms, and levels of inflammatory markers, such as C-reactive protein, are normal or slightly elevated (Khalil et al., 2019). Magnetic resonance imaging is needed to confirm the diagnosis, and if possible, at least two positive tissue cultures should be obtained. The major risk factor is a past history of spine surgery with an average of 34 months between the procedure and the onset of symptoms (Uçkay et al., 2010). In most cases, these patients have a favorable outcome with antibiotic therapy alone (Kowalski et al., 2007).

Certain controversy exists regarding the role of $C$. acnes in the pathogenesis of intervertebral disc degeneration and Modic type 1 changes of disc atrophy (fissuring and edema of the endplates) in herniated discs. In 2001, the study of Stirling et al. was the first to report positive cultures of $C$. acnes in intervertebral disc material after microdiscectomy (Stirling et al., 2001). Since then, several studies have reported the identification of $C$. acnes in patients with degenerative disc disease (Albert et al., 2013; Capoor et al., 2017, Ohrt-Nissen et al., 2018; Jha and Sairyo, 2020), although other studies do not support the true presence of C. acnes inside discs; if found it is often regarded as contaminant (Chen et al., 2016). Capoor et al. demonstrated the presence of a C. acnes biofilm in resected intervertebral discs by fluorescent in-situ hybridization (Capoor et al., 2017). In addition, Lin et al. showed that $C$. acnes may be involved in intervertebral disc degeneration by inducing apoptosis of nucleus pulposus cells via the TLR2/JNK pathway (Lin et al., 2018). The identification of $C$. acnes may indicate a true etiology in the pathogenesis of Modic type I changes, which could be responsive to antibiotic treatment, even though it is not possible to prove retrospectively which of those patients were truly infected. Further research and clinical trials are needed to clarify the infectious nature of $C$. acnes in this disease.

\section{Prostate Pathologies}

A recent review summarized the current evidence level of the involvement of $C$. acnes in prostate inflammation and prostate cancer (Brüggemann and Al-Zeer, 2020). There is relatively little doubt that $C$. acnes can be cultivated from a considerable portion of biopsy specimens, mainly obtained from radical prostatectomy. However, there is little knowledge about the exact origin of the recovered bacteria, i.e., if they represent contamination/carry-over from the urogenital tract, or if they are colonizers of the (anoxic regions) of the tumor tissue. For instance, a study from Sweden reported that $C$. acnes was cultured in $60 \%$ of the prostate cancer cases $(n=100)$ and in $26 \%$ of cancer-free controls $(n=50)$ (Davidsson et al., 2016). The phylotype II (SLST type K) was the most dominant type among C. acnes strains obtained from prostatic tissue and $26 \%$ of those strains carried an extrachromosomal element (Davidsson et al., 2017). In contrast, a French study has detected only very few C. acnes positive samples in their cohort $(n=36)$ (Bidaud et al., 2020); the biopsy procedure involved antibiotic prophylaxis that could explain the relative little bacterial recovery. Recent studies aimed at the determination of the urogenital tract (tumor) microbiome using culture-independent NGS-based techniques (Shrestha et al., 2018; Banerjee et al., 2019). Unfortunately, such low biomass studies often exclude $C$. acnes due to its notorious presence in control samples (Mollerup et al., 2016; Walker et al., 2020). Thus, NGS-based microbiome studies are unlikely to contribute significantly to the open questions regarding the presence and possible role of $C$. acnes in the prostate microenvironment.

\section{Sarcoidosis}

Sarcoidosis is a systemic inflammatory disease, characterized by the formation of non-necrotizing granulomas; these are most often detected in the lungs, but can be found also in the skin, lymph nodes, eyes, and other body sites and organs. The cause of sarcoidosis is unknown; infectious and noninfectious agents, individually or in combination, could play a role. Bacterial candidates as infectious causes of sarcoidosis are primarily Mycobacterium tuberculosis and C. acnes (Eishi, 2013). A recent review summarizes current insights in the disease contribution of $M$. tuberculosis and C. acnes to sarcoidosis (Yamaguchi et al., 2021). Several recent studies have identified $C$. acnes in granulomas of sarcoidosis patients; detection was achieved with DNA-based techniques, but also with immunohistochemistry/immunofluorescence staining (Nagata et al., 2017; Suzuki et al., 2018; Beijer et al., 2021). So far, no specific $C$. acnes phylotype has been associated with sarcoidosis. The involvement of $C$. acnes in sarcoidosis is also supported by experiments in mice (Werner et al., 2017; Song et al., 2019). As a possible disease scenario, invasive $C$. acnes, possibly derived from the skin, could result in a latent, intracellular state, for example in macrophages. Intracellular presence and/or proliferation could activate immune responses, mediated by bacterial factors, followed by the formation of insoluble immune complexes, in particular in individuals predisposed with a Th1 hypersensitivity against $C$. acnes (Yamaguchi et al., 2021). The multifaceted interactions of $C$. acnes with the immune system 
and relevant machineries such as autophagy are presented in the chapter Sensing and Signalling of Extra- and Intracellular $C$. acnes and Innate and Adaptive Immune Responses.

\section{C. acnes Virulence Factors and Traits}

A few bacterial products and traits that possibly could influence disease pathologies have been investigated. These include e.g., CAMP (Christie-Atkins-Munch-Petersen) factors, dermatansulfate adhesins (DsAs), lipases, sialidases, hyaluronidases, putative endoglycoceramidases, porphyrins, short-chain fatty acids (SCFAs), cell wall polysaccharide/lipoglycan, and lipoproteins (Brüggemann et al., 2004; Valanne et al., 2005; Lodes et al., 2006; Holland et al., 2010; Mak et al., 2013; Yu et al., 2016; Brüggemann, 2019; McLaughlin et al., 2019). Some of these factors are actively or passively secreted, others are part of the cell surface or attached to/embedded into the cell wall. A recent study has analyzed a sortase of $C$. acnes, an enzyme important for cell wall attachment of secreted proteins. The study predicted 19 sortase substrates, among them some of the above-mentioned factors, including CAMP factor 1 (locus tag: PPA1340), sialidases (PPA1560, PPA1821), and DsAs (PA2127, PPA2210) (Girolamo et al., 2019).

The evidence level of the involvement of the above-mentioned factors in disease pathology is incomplete. In fact, a clearcut virulence factor of $C$. acnes has yet to be identified, since there is very little insight into their functionalities. The lack of functional data can be partially explained with the lack of an efficient gene knock-out system for C. acnes; so far only very few mutant strains of $C$. acnes were created (Sörensen et al., 2010; Allhorn et al., 2016; Nazipi et al., 2017). Another reason is the apparent redundancy of host-interacting factors; $C$. acnes possesses multiple copies of genes for CAMP factors, DsAs, lipases, sialidases, putative endoglycoceramidases, and lipoproteins (Brüggemann et al., 2004). The redundancies indicate their importance for colonization and survival of $C$. acnes on human skin. There are only limited sequence differences regarding the mentioned host-interacting factors between the different $C$. acnes phylotypes. As an exception, substantial sequence differences can be detected for the hyaluronidase: there are two variants present in C. acnes. One variant is present in phylotype IA strains and the other variant is found in strains of phylotypes IB and II (Nazipi et al., 2017).

Here, we will briefly introduce and discuss new data regarding bacterial factors and traits that are suspected to be important for bacterial pathogenesis, in particular in $\mathrm{AV}$, namely the CAMP factors, lipases and the metabolites/molecules SCFAs and porphyrins. We will also briefly discuss the evidence for biofilm formation of $C$. acnes, and its relevance in IAIs.

\section{CAMP Factors}

A review has summarized knowledge regarding CAMP factors in C. acnes (McDowell et al., 2013). There are five CAMP factor homologs (CAMP1-CAMP5) present in (all phylotypes of) $C$. acnes. In particular, CAMP1 and CAMP2 are produced in higher amounts in most $C$. acnes strains. There are phylotype-specific variations; for example (some strains of) phylotype IB produce higher amounts of CAMP4 (Holland et al., 2010). CAMP2 and
CAMP4 are abundantly secreted, as well as CAMP1; however, CAMP1 is also cell surface-exposed; it is predicted to be a sortase substrate (Girolamo et al., 2019). In human follicular casts CAMP1 was the most abundantly detected CAMP factor, followed by CAMP2 (Bek-Thomsen et al., 2014). The redundancy of CAMP factors is intriguing. C. acnes not only secretes high amount of CAMP factors but also decorates its surface with this protein. Thus, it can be assumed that CAMP factors have a vital function in the biology and/or host-interaction of $C$. acnes.

Regarding their functionality, CAMP factors are described as co-hemolysins, based on in vitro assays in which sheep blood erythrocytes are used (Christie et al., 1944). However, the in vivo function is likely to be quite different. Functional studies on CAMP factors of group B streptococci indicate that they are able to form micropores in eukaryotic membranes (Lang and Palmer, 2003). For the CAMP2 of C. acnes it was shown that is has cytotoxic effects in keratinocytes and macrophages if applied as recombinant protein (Nakatsuji et al., 2011). Another study reported that Toll-like receptor 2 (TLR-2) can recognize CAMP1 (Lheure et al., 2016). If confirmed, CAMP1 could be a trigger for an innate immune response when $C$. acnes comes in close contact with human (immunocompetent) cells. However, since $C$. acnes colonization of human skin usually does not go along with any detectable inflammation, the suspected adverse effects of CAMP factors, i.e., cytotoxicity and innate immune activation, must be tightly inhibited in vivo. The true in vivo function of CAMP factors might be different; e.g., it could be envisaged that CAMP factors, due to their pore-forming activity, could be involved in accessing nutrients within the sebaceous follicle microenvironment, without causing excessive damage to intact keratinocytes.

\section{Lipases of C. acnes and Interaction With Host Glycolipids}

Sebaceous glands produce sebum, a mixture of relatively nonpolar lipids, in particular triglycerides, squalene, wax- and cholesterol-esters as well as free cholesterol. Sebum is usually overproduced during puberty due to the influence of androgens and other hormones on the sebaceous gland activity.

C. acnes has lipolytic properties; the bacterium possesses several lipases that can hydrolase triglycerides into fatty acids. A secreted lipase, the triacylglycerol lipase GehA, has been identified (Miskin et al., 1997). Interestingly, in human sebaceous follicles, another secreted lipase of $C$. acnes was detected in the infundibulum in larger amounts than GehA; this lipase was designated GehB (Bek-Thomsen et al., 2014). GehA and GehB are $42 \%$ identical on protein level.

The products of the activity of GehA/GehB, free fatty acids, have been suspected to contribute to acne. It is thought that alterations of the lipid composition in the infundibulum of the sebaceous follicle can provoke inflammatory responses (Zouboulis et al., 2014).

In addition to the hydrolysis of lipids, C. acnes possesses also several enzymes that can process glycolipids. Two putative endoglycoceramidases of $C$. acnes were identified within the infundibulum of sebaceous follicles (Bek-Thomsen et al., 2014). The proteins are predicted to catalyse the hydrolysis of the 
glycosidic linkage between oligosaccharides and ceramides of glycosphingolipids. As judged from the arsenal of enzymes that C. acnes possess, it can be hypothesized that the microorganism can process glycosphingolipids (e.g., gangliosides) to gain access to carbohydrates as nutrients: the oligosaccharide is removed from the ceramide by endoglycoceramidases; terminal sialic acid residues are removed by at least two sialidases (Brüggemann et al., 2004; Nakatsuji et al., 2008); the oligosaccharide moiety is further degraded by $\beta$-galactosidase, $\beta$-N-acetylhexosaminidase, endo- $\beta-N$-acetylglucosaminidase, and endo- $\alpha-N$-acetylglucosaminidase. Only the latter enzyme, designated EngPA was characterized so far (Koutsioulis et al., 2008).

\section{Short-Chain Fatty Acids (SCFAs)}

Short-chain fatty acids are metabolites produced by $C$. acnes during fermentative growth; propionate, but also acetate, butyrate and valerate have been reported to be produced by $C$. acnes, in particular in the presence of glycerol in the growth medium (Shu et al., 2013b; Sanford et al., 2016; Tax et al., 2016). Diverse roles have been associated with the production of SCFAs by C. acnes. Shu et al. and Wang et al. reported that SCFAs can suppress the growth of $S$. aureus (Shu et al., 2013b; Wang et al., 2014a), indicating that C. acnes can prevent the colonization of $S$. aureus on the skin via the production of SCFAs. Nakamura et al. reported that SFCAs produced by C. acnes can inhibit biofilm formation by $S$. epidermidis by an unknown mechanism (Nakamura et al., 2020). Sanford et al. reported that SCFAs of $C$. acnes might have an adverse effect for skin barrier function: SCFAs (mainly produced under hypoxic conditions in the presence of lipids) inhibited histone deacetylase (HDAC) activity in keratinocytes; as a consequence, cytokine production in those keratinocytes was elevated in response to TLR2 ligands (Sanford et al., 2016). A similar observation was made in sebocytes (Sanford et al., 2019). In contrast, SCFAmediated HDAC inhibition had the opposite consequence in monocytes (Sanford et al., 2016). Taken together, there seem to be health-beneficial as well as -detrimental roles of C. acnesproduced SFCAs. It is currently unknown, if this potentially fragile balance is influenced by the amount and/or the exact composition of SFCAs produced.

\section{Porphyrins}

C. acnes produce porphyrins as part of the vitamin B12 (cobalamin) cofactor biosynthesis pathway. They need cobalamin for essential enzymatic activities, e.g., for the activity of the methylmalonyl-CoA mutase, a key enzyme in the WoodWerkman cycle that yields propionate production. Depending on the growth conditions and possibly depending on the individual strain, different precursor molecules can be accumulated during cobalamin synthesis, such as protoporphyrin IX, uroporphyrin III, and coproporphyrin III (Shu et al., 2013a). Under anaerobic conditions, protoprophyrin IX seems dominant, whereas under aerobic conditions, coproporphyrin III is more abundant. The amount of produced porphyrins might be strain-dependent: in general, type I strains produced more porphyrins compared to type II and type III strains (Johnson et al., 2016; Barnard et al., 2020). Porphyrins are suspected to have multiple effects: as an adverse effect, coproporphyrin III can induce $S$. aureus aggregation and possibly biofilm formation (Wollenberg et al., 2014). Others reported that coproporphyrin III can trigger cytokine responses in exposed cells (Schaller et al., 2005). However, clear mechanisms have not been unraveled so far. Among many open questions, it will be interesting to investigate the role of $C$. acnes' porphyrins on other members of the skin microbiome.

\section{Biofilm}

One of the main virulence traits associated with IAIs is biofilm formation of the respective pathogen in opportunistic infections (Tande and Patel, 2014). Several studies have proven the ability of $C$. acnes to develop a biofilm in vitro, using a range of materials (Bayston et al., 2007; Tafin et al., 2012) and in different types of implants such as hip or knee prosthesis, sternal wires (Holmberg et al., 2009) or cardiac pacemaker devices (Okuda et al., 2018). Also in AV, biofilm formation of C. acnes has been proposed as a mechanism that allows bacterial persistency and partial resistance to antimicrobial therapy (Coenye et al., 2007; Spittaels and Coenye, 2018; Aslan Kayiran et al., 2020). A C. acnes biofilm was also observed in vivo in sebaceous follicles with distinct $C$. acnes colonization patterns, such as the attachment of C. acnes to the follicular wall or matrix-encased biofilms localized in the infundibulum of the follicle (Jahns and Alexeyev, 2014). It should be noted here that $C$. acnes (biofilm) could not be detected in intact sebaceous glands (Alexeyev and Jahns, 2012; Jahns and Alexeyev, 2014). Scanning electron microscopy studies also revealed the formation of biofilm ex vivo on implants such as shunts (Bayston et al., 2007) or intraocular lenses (Suzuki et al., 2020b). A few studies about C. acnes biofilm formation in animal models were performed. Tafin et al. developed a foreign bodyinfection model in guinea pigs and results showed the ability of $C$. acnes to adhere to the implant surface. This study also demonstrated that rifampin was the most effective antimicrobial agent against sessile $C$. acnes (Tafin et al., 2012). As also proven for other biofilm-forming bacteria, sessile $C$. acnes cells seem more resistant to antimicrobials than planktonic cells; this could explain why $C$. acnes-associated IAIs often respond insufficiently to antibiotic therapy alone (Coenye et al., 2007; Tafin et al., 2012). Shiono et al. showed that $C$. acnes could survive in a biofilm for at least 6 months, causing delayed surgical site infection in a mouse osteomyelitis model (Shiono et al., 2016). An IAI rabbit model was developed by Achermann et al. (2015). The study also identified proteins of $C$. acnes, produced when grown in a biofilm and as planktonic cells. The protein signature of C. acnes in both states was similar; identified proteins were mainly from the core metabolism and some stress-related factors. This indicates that there is no large reprogramming taking place when $C$. acnes switches from a planktonic to a biofilm-embedded, sessile state. However, Coeyne et al. observed increased lipase production in sessile bacteria compared to planktonic cells (Coenye et al., 2007).

The biofilm-embedment may allow $C$. acnes to exist in a latent, dormant state that does not provoke a substantial inflammatory host response. However, specific mechanisms employed by $C$. acnes that would allow the formation and maturation of a biofilm 
have not been investigated in detail. Kuehnast et al. proposed that there might be a correlation between biofilm formation and the C. acnes phylotype, rather than the anatomical site of infection (Kuehnast et al., 2018). The study showed that strains belonging to phylotype $\mathrm{IA}_{1}$ formed higher amounts of biofilm in microtiter plates than other phylotypes. This is in accordance with Okuda et al.; they demonstrated that biochemical properties and structures of biofilms differed among C. acnes isolates (Okuda et al., 2018). In addition, it was suggested that extracellular DNA may play a role in the formation of $C$. acnes biofilm, as also seen for many biofilms produced by other bacteria. Gannesen et al. develop a method to analyze the composition of the biofilm matrix of $C$. acnes. They found that the major polysaccharide component of the matrix was the same as the cell wall polysaccharide (Gannesen et al., 2019). This indicates that C. acnes does not produce a specific extracellular polysaccharide, but rather aggregates via the existing cell wall polysaccharide.

Taken together, the current data suggest that $C$. acnes can form biofilms or aggregates. However, $C$. acnes seems not to be a dedicated, specialized biofilm-former, i.e., it does not use a specific program to form a differentiated biofilm, like it is seen in other species such as ica-positive staphylococci. Indeed, compared to other biofilm-forming bacteria, C. acnes biofilms are rather weak and easily to dissolve, at least in vitro (Kuehnast et al., 2018). Observed differences in biofilm formation in different studies may, at least in part, be related to variations in the employed biofilm detection method and materials used rather than represent real biological variation (Holmberg et al., 2009). It seems likely that $C$. acnes uses endogenous molecules for adhesive purposes, whose main functions are related to structural integrity, e.g., cell wall polysaccharide. Biofilm formation in vivo can be fortified by host-derived molecules such as fibrinogen, as C. acnes has fibrinogen-binding proteins (DsAs) on its surface (Grange et al., 2017; Petersson et al., 2018; Pickering et al., 2019).

\section{Antimicrobial Resistance of C. acnes}

Antimicrobial resistance (AR) of $C$. acnes is a major concern in acne but also in other $C$. acnes-associated diseases. A few reviews have summarized the current knowledge regarding the types of $\mathrm{AR}$ and the dissemination of resistant $C$. acnes strains (Eady et al., 2003; Dessinioti and Katsambas, 2017; Karadag et al., 2021). In brief, there is a large variation concerning the frequency of AR of $C$. acnes in different countries, which might be due to different treatment strategies, in particular regarding acne. In general, higher frequencies of AR are observed regarding erythromycin and clindamycin, while resistance to tetracyclines is usually lower. Long-term use of antibiotics is associated with increased macrolide resistance (Nakase et al., 2018). Resistance in C. acnes to relevant antibiotics is mainly due to point mutations in genes encoding ribosomal RNAs (16S rRNA and 23S rRNA genes; Lomholt and Kilian, 2014; Nakase et al., 2017). In addition, erm $(\mathrm{X})$, encoding a RNA methylase that mediates resistance to clindamycin, has been identified on a mobile genetic element (Ross et al., 2002). Interestingly, a novel mobile genetic element of $C$. acnes was recently identified, conferring resistance to macrolides, clindamycin, and tetracyclines: the plasmid pTZC1 carries a novel macrolide-clindamycin resistance gene, erm(50), as well as a tetracycline resistance gene, tet $(\mathrm{W})$ (Aoki et al., 2020). Resistant strains of $C$. acnes often belong to the acneassociated types, i.e., phylotypes IA $_{1}$ (SLST types A and C) and IA $_{2}$ (SLST type F) (Lomholt and Kilian, 2014; Nakase et al., 2017; Sheffer-Levi et al., 2020).

\section{Sensing and Signaling of Extra- and Intracellular C. acnes and Innate and Adaptive Immune Responses}

C. acnes interacts on various levels with human cells such as skinresident keratinocytes, but possibly also with sebocytes and with immune cells, including skin-resident Langerhans cells (LCs), dendritic cell (DCs), macrophages, and $\mathrm{T}$ cells (reviewed in Mayslich et al., 2021). Previous studies have shown, mostly in cell culture, that $C$. acnes has extensive immunostimulatory activity. The bacterium can stimulate the production of antimicrobial peptides and diverse chemokine and cytokines. For instance, in keratinocytes, activation of hBD2, TNF- $\alpha$, GM-CSF, and IL- $1 \alpha$, IL-1 $\beta$, and IL- 8 has been observed upon bacterial encounter; in monocytes, $C$. acnes triggers the production of cytokines such as IL-1 $\beta$, IL-8, IL-12, and TNF- $\alpha$ (Chen et al., 2002; Nagy et al., 2005; Yu et al., 2016). Innate host cell receptors can sense C. acnes, either extra- or intracellularly. Important for sensing extracellular bacteria is TLR-2, since TLR-2 activation is crucial for NF- $\kappa \mathrm{B}$ activation in response to C. acnes (Kim et al., 2002; Su et al., 2017). As a TLR-2 agonist, CAMP factor 1 of C. acnes might play a role (Lheure et al., 2016). Intracellular recognition of $C$. acnes possibly involves other receptors, such as TLR-9 and NOD-like receptors, in a cell type-dependent manner (Kalis et al., 2005; Tanabe et al., 2006). In addition, C. acnes can activate the NLRP3 inflammasome, a system responsible for the activation of inflammatory processes via IL- $1 \beta$ maturation (Kistowska et al., 2014; Qin et al., 2014). A recent study has shown the involvement of another pathway in sensing intracellular $C$. acnes: bacterial DNA seems to be sensed in the host cell cytosol via the cGAS/STING pathway, resulting in a type I interferon response (Fischer et al., 2020). C. acnes likely interacts also with other immune cells such as T-cells. The bacterium can induce the production of T helper type 1(Th1)-type cytokines (e.g., IL-12, IFN- $\gamma$, and TNF- $\alpha$ ) and Th17-type cytokines (e.g., IL-17 and IL22) (Thielitz et al., 2008; Agak et al., 2014; Kistowska et al., 2015). In line with this, C. acnes-specific Th17 and Th1/Th17 cells can be detected in the peripheral blood of acne patients. Different $C$. acnes strains seem to have distinguishable abilities, for example to induce Th17-type cytokines; thus, C. acnes might modulate $\mathrm{CD}^{+}$T-cell responses in a strain-specific manner (Agak et al., 2018).

Taken together, the pro-inflammatory activity of $C$. acnes involves host cell signaling pathways and systems such as NF- $\kappa \mathrm{B}$, the inflammasome, and STING/cGAS. Less well-understood is which C. acnes components, e.g., genomic DNA, surface/secreted proteins, peptidoglycan and cell wall polysaccharide, are actually sensed and how the pro-inflammatory activity of $C$. acnes is controlled/dampened on normal skin. C. acnes-exposed keratinocytes may attenuate TLR-induced inflammation by negative regulatory circuits involving proteins such as TNIP1 
and TNFAIP3 and microRNAs such as miR-146a, that can downregulate the production of inflammatory cytokines and chemokines (Erdei et al., 2018, 2021; Zeng et al., 2019). In addition, besides keeping bacteria in physical distance to immunocompetent cells, e.g., within infundibula of sebaceous follicles, another strategy to limited responses might be to eliminate invasive $C$. acnes. Intracellular bacteria are degraded by endocytosis/phagocytosis and in addition, the autophagic pathway is involved (Nakamura et al., 2016; Megyeri et al., 2018). It was suggested that induction of autophagy is caused by $C$. acnes cells in the host cell cytosol that escaped from endosomes (Nakamura et al., 2016). Another open question concerns the extent and significance of strain differences in host cell responses; here, knowledge remains fragmentary, since different researchers use often different strains. Thus, a robust, multi-laboratory investigation is needed with a defined panel of well-characterized C. acnes strains.

\section{DR. JEKYLL-PROVEN AND SUGGESTED HEALTH-BENEFICIAL ROLES OF C. acnes}

\section{General Considerations}

Since the first time Antonie van Leeuwenhoek set his eyes on bacteria in 1683, the scientific community has focused on understanding their role in pathogenesis, deciphering the hostpathogen interactions through molecular analysis of virulence factors and traits. This focus has paved the way to medical land-winnings including vaccines, antibiotics, and the general idea of hygiene. While a focus on bacterial pathogens and their molecular mediators of virulence has lent us much knowledge and medical tools, it has also to a large extent made us forget about the majority of bacteria, living in symbiosis with us, being commensal or mutualistic in their interaction.

The idea of some bacteria being beneficial was raised several centuries ago, but has recently received increased attention (Fijan, 2014), and also turned more molecular (Mazmanian et al., 2008; Wang et al., 2014c; Wollein Waldetoft et al., 2020). Now it is well-recognized that (commensal) bacteria play a critical role in maintaining health and microbial dysbiosis being a cause to several common diseases (Harmsen and de Goffau, 2016; Zhu et al., 2018). We recognize the importance of gut bacteria for production of vital metabolic products (e.g., vitamin K; Conly and Stein, 1992) and how commensals produce bacteriocins and antibacterial substances in order to reduce the colonization of pathogens (Hammami et al., 2013), as well as how bacteria orchestrate the immune system to improve its defensive capacity (Naik et al., 2015). The raised awareness of beneficial aspects of the commensal microbiota has led to a reevaluation of some of the bacterial members of the microbiota. In particular, the ubiquitous skin bacterium C. acnes has received much attention during the last years, being suggested to not only limit colonization by more potent pathogens (Wang et al., 2014a), but also to positively modulate the immune system (da Silva et al., 2013; Talib and Saleh, 2015), produce beneficial metabolites (Christensen and Brüggemann, 2014), treat and protect from tumor development (Tsuda et al., 2011), and

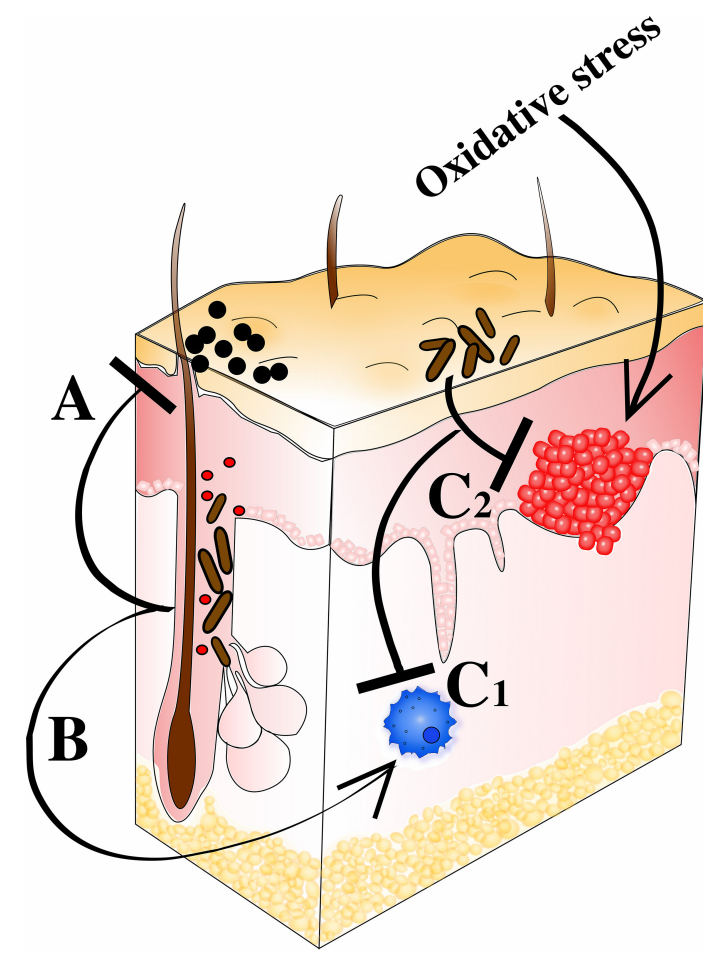

FIGURE 4 | C. acnes' prospective probiotic qualities. (A) Colonization resistance against staphylococci and other potential pathogens. Direct effects through expression of SCFAs (e.g., propionic acid) and bacteriocins (e.g., cutimycin). Indirect effects through induction of host-derived AMPs (e.g., HBD and LL-37). (B) Modulation of immune response including e.g., Th1 and Th17 priming leading to heightened intrinsic anti-tumor activity and clearance of pathogens. Interactions with Langerhans cells also help shape host immune defenses. (C1) Inflammation regulation via RoxP, CLAs, polysaccharides, and SCFAs (pro- and anti-inflammatory qualities). (C2) Counteracting the tumorigenic effect of oxidative stress through RoxP secretion.

maintain redox homeostasis on the skin (Allhorn et al., 2016). Here we summarize the documented beneficial effects of C. acnes, describe their molecular mechanisms, and discuss their impact in maintaining our health (Figure 4).

\section{C. acnes As a Regulator of Pathogenic Skin Colonizers}

The commensal microbiota has been attributed many generic functions, including the ability to limit colonization by more pathogenic bacteria (Abt and Pamer, 2014). While undoubtedly so, the molecular mechanisms underlying such inhibition are mostly unknown. Production of antibacterial substances, reduction of nutritional availability and steric hindrance to adhere to the host have been suggested to be important factors (Silva et al., 2015). C. acnes, colonizing a highly specific niche of the skin, employs several mechanisms to promote its exclusivity in this environment.

\section{Bacteriocins as Regulators of the Skin Microbiota}

Competition for colonization not only exists between different bacterial species, but also between different bacterial strains. For 
that purpose, several bacteria produce bacteriocins that can target similar bacteria. Two different bacteriocins have been reported for C. acnes; acnecin and a thiopeptide, designated cutimycin. Acnecin was identified 1978, but no follow-up studies have been conducted since. The protein was determined to be a pentamer consisting of five $12 \mathrm{kDa}$ monomers, exerting an antibacterial effect toward non-acnecin producing $C$. acnes strains, but not toward other species (Fujimura and Nakamura, 1978; Nakamura et al., 1978). Further characterizations concluded its protein composition, being negatively charged (pI 5.5) with a low carbohydrate content. Strikingly, acnecin was reported to be bacteriostatic only, not bactericidal (Fujimura and Nakamura, 1978).

The thiopeptide cutimycin has only recently been identified through comparative genomic studies (Brüggemann et al., 2012), uniquely found in type IB C. acnes strains only (Christensen et al., 2016). This peptide was shown to efficiently kill S. epidermidis and thus give $C$. acnes a competitive advantage to colonize the skin (Claesen et al., 2020). On a similar basis, S. epidermidis was also shown to be able to limit growth of $C$. acnes by several mechanisms and molecules; for example, fermentation products of $S$. epidermidis have been shown to mediate such reduction of fitness for C. acnes (Wang et al., 2014b). In contrast, Xia et al. demonstrated how $S$. epidermidis can locally reduce $C$. acnes induced inflammation, thus improving $C$. acnes fitness on the skin (Xia et al., 2016). This suggests that this microbe-microbe interaction is multifaceted.

\section{Acidification by Propionic Acid Creates a Niche for C. acnes}

A broader, less specific means to limit colonization by other bacterial species is mediated by the creation of highly specific niches. The former name of $C$. acnes, Propionibacterium acnes, relates to its ability to ferment sebum triglycerides and produce propionic acid. SCFAs, among them propionic acid, produced by C. acnes results in a lowered $\mathrm{pH}$ that is toxic to many microbes, both bacteria and yeast (Wang et al., 2014a). The ability of $C$. acnes to kill $S$. aureus through production of propionic acid has been proven both in vitro and in vivo (Shu et al., 2013b), and may partake in limiting $S$. aureus colonization on skin. Thereby, it was shown that the acidity per se is not toxic to the bacteria, but a high concentration of propionic acid itself $(>25 \mathrm{mM})$ is efficiently killing several species. C. acnes itself is resistant to the inhibitory effect of propionic acid at biologically relevant levels (Wang et al., 2014a), allowing it to create an ecological niche on the skin, free of (most) other microbial species. The ability to produce propionic acid and to acidify the surrounding has however also been associated with the pathogenicity of $C$. acnes (Tax et al., 2016), making it a dual-edged sword.

\section{Short Chain Fatty Acids Produced by C. acnes Promote a Healthy Microbiota}

Besides the ability of $C$. acnes to produce propionic acid from sebum components such as triglycerides, a few other SCFAs (acetic acid, butyric acid, and valeric acid) can also be produced through fermentation (Shu et al., 2013b; Sanford et al., 2016). SCFAs have proven to limit growth and colonization of several skin pathogens (e.g., S. aureus and Streptococcus pyogenes) while not significantly affecting the growth of skin commensals (Gribbon et al., 1993; Hevia et al., 2015). Besides serving as a mean to limit pathogen colonization, the presence of SCFAs entail increased metabolic opportunities for the host (Morrison and Preston, 2016; LeBlanc et al., 2017). Furthermore, such SCFAs have been proven to positively regulate both inflammation and development of cancer (LeBlanc et al., 2017). It has been argued that the ability of $C$. acnes to produce such substances is a positive trait of a mutualistic lifestyle, stressing its benevolent nature (Christensen and Brüggemann, 2014).

\section{Beneficial Immunomodulating Properties of C. acnes}

Since the discovery of C. acnes around 1,900 (Evans et al., 1950; Moore and Cato, 1963), its inflammatory and thereby immunomodulatory properties have been recognized. Initially seen as only a pathogenic trait of $C$. acnes, the induction of a Th1-type immune response has been taken advantage of for preventing, protecting, and treating several different pathologies.

\section{C. acnes As an Adjuvant Reduces Sensitivity to Infections by Other Microbes}

Due to the inflammatory properties of $C$. acnes, heat-killed C. acnes bacteria are commonly used as adjuvants in vaccines e.g., for horses, activating macrophages efficiently through its unique peptidoglycan structure (Peters and Hay, 1990; Paillot, 2013). However, injection of heat-killed C. acnes into a host has also been shown to affect sensitivity to infection by specific microbes as well as polymicrobial infections. While not being considered a zoonotic pathogen, the impact of Actinobacillus pleuropneumoniae on pig production is substantial ( $\mathrm{Li}$ et al., 2013). Pre-treatment with C. acnes leads to a potent defense against A. pleuropneumoniae infections, likely due to crossreactivity between one or several $C$. acnes antigens. A similar study in mice reached the same conclusion, showing a $C$. acnes dose-dependent protection toward A. pleuropneumoniae infection (Yang et al., 2014). Other studies have shown that pretreatment of mice with heat-killed $C$. acnes allows for a higher survival rate (50 vs. $0 \%$ ) when exposed to a polymicrobially induced sepsis (da Silva et al., 2013), indicating a more general priming of the immune system. Similarly, pre-treatment of mice with heat-killed $C$. acnes significantly improves infection with the parasite (worm) Heligmosomoides polygyrus (GonzálezSánchez et al., 2014). Thus, C. acnes may act as a general immunomodulator, orchestrating our immune defense against pathogens. In line with this, it was recently shown by Agak et al. how the presence of $C$. acnes can generate specific $\mathrm{T}_{\mathrm{H}} 17$ clones with a broad antibacterial activity, protecting against pathogens (Agak et al., 2021).

\section{C. acnes Can Treat Solid Tumors}

Due to the immunomodulatory effects of $C$. acnes, inducing a Th1 response, it was speculated if injections of $C$. acnes could shift Th2 responses toward Th1. A common Th2-driven disease is tumors, protecting themselves through modulating the immune response into a B-cell response (e.g., Th2) rather than a Th1-response 
that would be more apt to clear tumors through cytotoxic T-cells and NK cells (Disis, 2010). Historically, heat-killed C. acnes has been experimentally injected intra-tumorally in mice, resulting in significantly reduced pathology and tumor size (Bartlett et al., 1980; Kennedyl and Conley, 1989). Treatment of melanoma led to a switch in the immune response, both locally (skin) and systemically, to a Th1 response, resulting in heavy infiltration of T-cells to tumor lesions (Tsuda et al., 2011). In breast cancer a similar immunological change was observed, resulting in the complete removal of tumors in $40 \%$ of the mice, and significantly reduced angiogenesis while given in combination with melatonin (Talib and Saleh, 2015). With no reported toxicity and significant anti-tumor effect, the usage of $C$. acnes as an immunomodulator for tumor reduction may be a future therapy option.

\section{Th1-Driven Response to C. acnes Restores Skin Pathologies}

Several skin disorders have immunomodulatory pathologies, including atopic dermatitis and psoriasis (Griffiths et al., 2017). Both diseases have been reported to be associated with microbial dysbiosis, having significantly increased levels of $S$. aureus and reduced levels of C. acnes (Gao et al., 2008; Francuzik et al., 2018), and the presence of C. acnes has been suggested to play an important role in maintaining the skin health by influencing the skin microbiome (Chang et al., 2018). Supporting this claim, Kitagawa et al. demonstrated the protective effect of C. acnes, inducing a Th1 immune response locally and systemically, that resulted in significant improvement of atopic dermatitis in mice (Kitagawa et al., 2011). Similarly, the Th2-driven disease focal segmental glomerulosclerosis can be prevented, or limited if treated after onset, with the polysaccharide fraction of $C$. acnes (Reis et al., 2012). Whether the presence of C. acnes on the skin can protect from, or reduce the risk of, development of Th2driven skin diseases is currently unknown. However, the current data suggest that this may be an interesting scientific avenue to pursue.

\section{Health-Beneficial Molecules Produced by C. acnes}

Besides the abovementioned health-promoting aspects of $C$. acnes, several recent reports have indicated a direct health beneficial potential of $C$. acnes, by means of secreted molecules that may positively impact our health (Cogen et al., 2008). These benevolent factors may play a role in the protection from several diseases including oxidative skin diseases and skin cancer $(\mathrm{He}$ et al., 2015; Allhorn et al., 2016).

\section{Conjugated Linoleic Acid (CLA)}

Different variants of CLAs can have significant health-beneficial effects, including lowering cancer risks and enhanced immune defenses (Benjamin et al., 2015). Production of those acids are mainly through chemical reactions, resulting in a mixture of isomers, with some being more beneficial than others (Yang and Liu, 2004). However, it was recognized that C. acnes possesses a linoleic acid isomerase creating trans-10, cis-12 CLA as a single isomer (He et al., 2015) The biological relevance of the isomerase and the exact role of trans-10, cis-12 CLA on the skin have yet to be explored.

\section{Antioxidant RoxP}

Being a facultative anaerobe, $C$. acnes has developed several means to protect itself from oxidative stress, including catalase, peroxidases, and superoxide dismutase (Rolfe et al., 1978). Recently, it was discovered that $C$. acnes secrets high levels of the potent antioxidant RoxP. RoxP was found to be unique for $C$. acnes, conserved in all strains, and indispensable for an efficient colonization on skin (Allhorn et al., 2016). Due to an oxidative element in several skin pathologies the presence of $C$. acnes and RoxP could have protective functions and limit the negative effect of oxidative stress and development of disease.

Oxidation of skin, mediated by UVB irradiation, is a common cause of skin cancer (Narendhirakannan and Hannah, 2013). While C. acnes stimulate apoptosis of UVB-damaged melanocytes, other skin bacteria (e.g., S. epidermidis) promote their survivability (Talib and Saleh, 2015). Thus, not only may C. acnes prevent initial oxidative stress through secretion of antioxidants, but may also be capable of clearing cancerous skin cells.

Using RoxP as a biomarker for healthy skin, several highly sensitive biosensors have been developed (Ertürk and Lood, 2018; Ertürk et al., 2018; Ertürk Bergdahl et al., 2019), enabling absolute quantification of RoxP from skin samples from a diversity of skin conditions (healthy, actinic keratosis, basal cell cancer). This demonstrated the association of RoxP and C. acnes with oxidative diseases (Andersson et al., 2019). The ability of an ubiquitous skin bacterium to produce high quantities of an antioxidant, that aids in the protection from oxidative stress is of interest from both a biological and medical perspective. Modulation of the microbiota may thus be a means of affecting the redox homeostasis of the skin. Further studies are needed to properly evaluate the in vivo role of RoxP.

\section{CONCLUSIONS}

Originally identified from acne, and historically being disreputed as a skin pathogen only, more and more evidence has enabled us to start understanding the complex roles of C. acnes. Recent research indicates that this species is a beneficial skin bacterium that fulfills important roles for skin homeostasis and protection. It also became clear that host responses to $C$. acnes can have both, beneficial and detrimental consequences. The criteria that decide about the outcome are multifactorial, and include, among others, the C. acnes phylotype/strain composition and population size, the tissue location of $C$. acnes, host tissue/cell tropism, the interaction with other skin bacteria, the predisposition and status of the host, including host genetics, as well as possibly other factors that are currently poorly understood, such as the influence of the gut microbiota.

Regarding the involvement of $C$. acnes in disease, it is widely accepted that $C$. acnes plays an important role in skin disorders such as $\mathrm{AV}$, even though there are still gaps in our understanding of its exact contribution to disease. More and more studies point to a strong impact of the skin's immune system and the 
interactions of $C$. acnes with skin-resident immunocompetent cells. Thus, in order to understand the role of $C$. acnes in $\mathrm{AV}$, one needs to understand the immune system of the skin.

Regarding the involvement of $C$. acnes in non-skin diseases there is a more controversial debate. One reason for the skepticism is connected to the omnipresence of $C$. acnes, which makes it difficult/impossible to apply Koch's postulates. It is difficult to exclude skin-derived contamination during sampling of the diseased tissue site; in this regard, DNA-based diagnostic tools applied on human specimens may be oversensitive, in particular regarding studies that use samples (e.g., tissue biopsies) with low microbial biomass (Eisenhofer et al., 2019). Thus, despite intensive research, our knowledge about the contribution of $C$. acnes to non-skin diseases is far from being complete. For IAIs, there has been an opinion change in the last decade; the scientific community now largely acknowledges that $C$. acnes can be a monomicrobial cause of IAIs, like other skinresident bacteria such as coagulase-negative staphylococci. In Table 1 we have tried to evaluate the current evidence level for a (major) role of $C$. acnes in several diseases, based on clinical and experimental data.

Further understanding of C. acnes' biology is needed; in particular, we need to know more about its produced and secreted factors and their host-interacting functions in order to enable

\section{REFERENCES}

Abt, M. C., and Pamer, E. G. (2014). Commensal bacteria mediated defenses against pathogens. Curr. Opin. Immunol. 29, 16-22. doi: 10.1016/j.coi.2014.03.003

Achermann, Y., Goldstein, E. J. C., Coenye, T., and Shirtliffa, M. E. (2014). Propionibacterium acnes: from Commensal to opportunistic biofilm-associated implant pathogen. Clin. Microbiol. Rev. 27, 419-440. doi: 10.1128/CMR.00092-13

Achermann, Y., Tran, B., Kang, M., Harro, J. M., and Shirtliff, M. E. (2015). Immunoproteomic identification of in vivo-produced Propionibacterium acnes proteins in a rabbit biofilm infection model. Clin. Vaccine Immunol. 22, 467-476. doi: 10.1128/CVI.00760-14

Agak, G. W., Kao, S., Ouyang, K., Qin, M., Moon, D., Butt, A., et al. (2018). Phenotype and antimicrobial activity of Th17 cells induced by Propionibacterium acnes strains associated with healthy and acne skin. J. Investig. Dermatol. 138, 316-324. doi: 10.1016/j.jid.2017.07.842

Agak, G. W., Mouton, A., Teles, R. M. B., Weston, T., Morselli, M., Andrade, P. R., et al. (2021). Extracellular traps released by antimicrobial TH17 cells contribute to host defense. J. Clin. Investig. 131:JCI141594. doi: 10.1172/JCI141594

Agak, G. W., Qin, M., Nobe, J., Kim, M. H., Krutzik, S. R., Tristan, G. R., et al. (2014). Propionibacterium acnes induces an IL-17 response in acne vulgaris that is regulated by vitamin A and vitamin D. J. Investig. Dermatol. 134, 366-373. doi: 10.1038/jid.2013.334

Albert, H. B., Lambert, P., Rollason, J., Sorensen, J. S., Worthington, T., Pedersen, M. B., et al. (2013). Does nuclear tissue infected with bacteria following disc herniations lead to Modic changes in the adjacent vertebrae? Eur. Spine J. 22, 690-696. doi: 10.1007/s00586-013-2674-z

Alexeyev, O. A., Dekio, I., Layton, A. M., Li, H., Hughes, H., Morris, T., et al. (2018). Why we continue to use the name Propionibacterium acnes. Br. J. Dermatol. 179:1227. doi: 10.1111/bjd.17085

Alexeyev, O. A., and Jahns, A. C. (2012). Sampling and detection of skin Propionibacterium acnes: current status. Anaerobe 18, 479-483. doi: 10.1016/j.anaerobe.2012.07.001

Alexeyev, O. A., Marklund, I., Shannon, B., Golovleva, I., Olsson, J., Andersson, C., et al. (2007). Direct visualization of Propionibacterium acnes in prostate us to better recognize the beneficial and detrimental aspects of the bacterium and take advantage of them for improving (skin) health.

\section{AUTHOR CONTRIBUTIONS}

$\mathrm{HB}$ and RL wrote the main parts of the manuscript. LS-V and HG wrote specific sections. All authors contributed to the article and approved the submitted version.

\section{FUNDING}

This work was supported by grants from the Royal Physiographic Society of Lund, The Swedish Society for Medicine, the NovoNordisk Foundation (NNF18OC0053172), and the foundations of Crafoord, Längmanska Kulturfonden, Alfred Österlund, Tornspiran, Magnus Bergvall, Åke Wiberg, Clas Groschinsky, and Sigurd and Elsa Goljes Minne.

\section{ACKNOWLEDGMENTS}

Tilde Andersson was acknowledged for excellent and appreciated assistance with figure design. tissue by multicolor fluorescent in situ hybridization assay. J. Clin. Microbiol. 45, 3721-3728. doi: 10.1128/JCM.01543-07

Allhorn, M., Arve, S., Brüggemann, H., and Lood, R. (2016). A novel enzyme with antioxidant capacity produced by the ubiquitous skin colonizer Propionibacterium acnes. Sci. Rep. 6:srep36412. doi: 10.1038/srep36412

Andersson, T., Ertürk Bergdahl, G., Saleh, K., Magnúsdóttir, H., Stødkilde, K., Andersen, C. B. F., et al. (2019). Common skin bacteria protect their host from oxidative stress through secreted antioxidant RoxP. Sci. Rep. 9:3. doi: 10.1038/s41598-019-40471-3

Aoki, S., Nakase, K., Nakaminami, H., Wajima, T., Hayashi, N., and Noguchi, N. (2020). Transferable multidrug-resistance plasmid carrying a novel macrolideclindamycin resistance gene, erm(50), in Cutibacterium acnes. Antimicrob. Agents Chemother. 64, e01810-e01819. doi: 10.1128/AAC.01810-19

Aslan Kayiran, M., Karadag, A. S., Al-Khuzaei, S., Chen, W., and Parish, L. C. (2020). Antibiotic resistance in acne: mechanisms, complications and management. Am. J. Clin. Dermatol. 21, 813-819. doi: 10.1007/s40257-020-00556-6

Asseray, N., Papin, C., Touchais, S., Bemer, P., Lambert, C., Boutoille, D., et al. (2010). Improving diagnostic criteria for Propionibacterium acnes osteomyelitis: a retrospective analysis. Scand. J. Infect. Dis. 42, 421-425. doi: 10.3109/00365540903527330

Aubin, G. G., Lavigne, J. P., Foucher, Y., Dellière, S., Lepelletier, D., Gouin, F., et al. (2017). Tropism and virulence of Cutibacterium (formerly Propionibacterium) acnes involved in implant-associated infection. Anaerobe 47, 73-78. doi: 10.1016/j.anaerobe.2017.04.009

Aubin, G. G., Portillo, M. E., Trampuz, A., and Corvec, S. (2014). Propionibacterium acnes, an emerging pathogen: from acne to implantinfections, from phylotype to resistance. Med. Mal. Infect. 44, 241-250. doi: 10.1016/j.medmal.2014.02.004

Bae, Y., Ito, T., Iida, T., Uchida, K., Sekine, M., Nakajima, Y., et al. (2014). Intracellular propionibacterium acnes infection in glandular epithelium and stromal macrophages of the prostate with or without cancer. PLOS ONE 9:324. doi: 10.1371/journal.pone.0090324

Banerjee, S., Alwine, J. C., Wei, Z., Tian, T., Shih, N., Sperling, C., et al. (2019). Microbiome signatures in prostate cancer. Carcinogenesis 40, 749-764. doi: $10.1093 /$ carcin/bgz008 
Banzon, J. M., Rehm, S. J., Gordon, S. M., Hussain, S. T., Pettersson, G. B., and Shrestha, N. K. (2017). Propionibacterium acnes endocarditis: a case series. Clin. Microbiol. Infection 23, 396-399. doi: 10.1016/j.cmi.2016. 12.026

Barnard, E., Johnson, T., Ngo, T., Arora, U., Leuterio, G., McDowell, A., et al. (2020). Porphyrin production and regulation in cutaneous Propionibacteria. mSphere 5:19. doi: 10.1128/mSphere.00793-19

Barnard, E., Liu, J., Yankova, E., Cavalcanti, S. M., Magalhães, M., Li, H., et al. (2016). Strains of the Propionibacterium acnes type III lineage are associated with the skin condition progressive macular hypomelanosis. Sci. Rep. 6:srep31968. doi: 10.1038/srep31968

Bartlett, G. L., Kreider, J. W., and Purnell, D. M. (1980). Treatment of cancer using Corynebacterium parvum: similarity of two preparations in four animal tumor models. Cancer 46, 685-691. doi: 10.1002/10970142(19800815)46:4<685::AID-CNCR2820460409>3.0.CO;2-T

Bayston, R., Ashraf, W., Barker-Davies, R., Tucker, E., Clement, R., Clayton, J., et al. (2007). Biofilm formation by Propionibacterium acnes on biomaterials in vitro and in vivo: impact on diagnosis and treatment. J. Biomedical Mater. Res. A 81, 705-709. doi: 10.1002/jbm.a.31145

Bayston, R., Vera, L., and Ashraf, W. (2010). Activity of an antimicrobial hydrocephalus shunt catheter against Propionibacterium acnes. Antimicrob. Agents Chemotherapy 54, 5082-5085. doi: 10.1128/AAC.00540-10

Beijer, E., Seldenrijk, K., Eishi, Y., Uchida, K., Damen, J., Grutters, J. C., et al. (2021). Presence of Propionibacterium acnes in granulomas associates with a chronic disease course in Dutch sarcoidosis patients. ERJ Open Res. 7, 486-2020. doi: 10.1183/23120541.00486-2020

Bek-Thomsen, M., Lomholt, H. B., Scavenius, C., Enghild, J. J., and Brüggemann, H. (2014). Proteome analysis of human sebaceous follicle infundibula extracted from healthy and acne-affected skin. PLOS ONE 9:107908. doi: 10.1371/journal.pone.0107908

Bémer, P., Corvec, S., Tariel, S., Asseray, N., Boutoille, D., Langlois, C., et al. (2008). Significance of Propionibacterium acnes-positive samples in spinal instrumentation. Spine 33:2008. doi: 10.1097/BRS.0b013e31818e28dc

Benito, N., Mur, I., Ribera, A., Soriano, A., Rodríguez-Pardo, D., Sorlí, L., et al. (2019). The different microbial etiology of prosthetic joint infections according to route of acquisition and time after prosthesis implantation, including the role of multidrug-resistant organisms. J. Clin. Med. 8:673. doi: 10.3390/jcm8050673

Benjamin, S., Prakasan, P., Sreedharan, S., Wright, A. D. G., and Spener, F. (2015). Pros and cons of CLA consumption: an insight from clinical evidences. Nutr. Metab. 12:4. doi: 10.1186/1743-7075-12-4

Bidaud, A. L., Karam, G., Kandel-Aznar, C., Ruffier d'Epenoux, L., Guillouzouic, A., Bémer, P., et al. (2020). Low prevalence of Cutibacterium acnes in prostatic tissue biopsies in a French hospital. Anaerobe 66:102286. doi: $10.1016 /$ j.anaerobe.2020.102286

Bocquet-Trémoureux, S., Corvec, S., Khammari, A., Dagnelie, M. A., Boisrobert, A., and Dreno, B. (2020). Acne fulminans and Cutibacterium acnes phylotypes. J. Eur. Acad. Dermatol. Venereol. 34, 827-833. doi: 10.1111/jdv.16064

Boisrenoult, P. (2018). Cutibacterium acnes prosthetic joint infection: diagnosis and treatment. Orthopaed. Traumatol. Surg. Res. 104, S19-S24. doi: 10.1016/j.otsr.2017.05.030

Bossard, D. A., Ledergerber, B., Zingg, P. O., Gerber, C., Zinkernagel, A. S., Zbinden, R., et al. (2016). Optimal length of cultivation time for isolation of Propionibacterium acnes in suspected bone and joint infections is more than 7 days. J. Clin. Microbiol. 54, 3043-3049. doi: 10.1128/JCM.01435-16

Brüggemann, H. (2019). "Skin: Cutibacterium (formerly Propionibacterium) acnes and Acne Vulgaris," in Health Consequences of Microbial Interactions with Hydrocarbons, Oils, and Lipids, ed H. Goldfine (Springer International Publishing), 1-20. doi: 10.1007/978-3-319-72473-7_20-1

Brüggemann, H., and Al-Zeer, M. A. (2020). Bacterial signatures and their inflammatory potentials associated with prostate cancer. APMIS 128:13021. doi: 10.1111/apm.13021

Brüggemann, H., Henne, A., Hoster, F., Liesegang, H., Wiezer, A., Strittmatter, A., et al. (2004). The complete genome sequence of Propionibacterium acnes, a commensal of human skin. Science 305, 671-673. doi: 10.1126/science.1100330

Brüggemann, H., Lomholt, H. B., and Kilian, M. (2012). The flexible gene pool of Propionibacterium acnes. Mobile Genetic Elements 2, 145-148. doi: $10.4161 /$ mge. 21204
Burnham, J. P., Shupe, A., Burnham, C. A. D., and Warren, D. K. (2017) Utility of strain typing of Propionibacterium acnes in central nervous system and prosthetic joint infections to differentiate contamination from infection: a retrospective cohort. Eur. J. Clin. Microbiol. Infect. Dis. 36, 2483-2489. doi: 10.1007/s10096-017-3090-9

Byrd, A. L., Belkaid, Y., and Segre, J. A. (2018). The human skin microbiome. Nat. Rev. Microbiol. 16, 143-155. doi: 10.1038/nrmicro.2017.157

Capoor, M. N., Birkenmaier, C., Wang, J. C., McDowell, A., Ahmed, F. S. Brüggemann, H., et al. (2019). A review of microscopy-based evidence for the association of Propionibacterium acnes biofilms in degenerative disc disease and other diseased human tissue. Eur. Spine J. 28, 2951-2971. doi: 10.1007/s00586-019-06086-y

Capoor, M. N., Ruzicka, F., Schmitz, J. E., James, G. A., Machackova, T., Jancalek, R., et al. (2017). Propionibacterium acnes biofilm is present in intervertebral discs of patients undergoing microdiscectomy. PLoS ONE 12:174518. doi: 10.1371/journal.pone.0174518

Chang, H. W., Yan, D., Singh, R., Liu, J., Lu, X., Ucmak, D., et al. (2018). Alteration of the cutaneous microbiome in psoriasis and potential role in Th17 polarization. Microbiome 6:1. doi: 10.1186/s40168-018-0533-1

Chen, Q. J., Koga, T., Uchi, H., Hara, H., Terao, H., Moroi, Y., et al. (2002). Propionibacterium acnes-induced IL-8 production may be mediated by NF-кB activation in human monocytes. J. Dermatol. Sci. 29, 97-103. doi: 10.1016/S0923-1811(02)00013-0

Chen, Z., Cao, P., Zhou, Z., Yuan, Y., Jiao, Y., and Zheng, Y. (2016). Overview: the role of Propionibacterium acnes in nonpyogenic intervertebral discs. Int. Orthop. 40, 1291-1298. doi: 10.1007/s00264-016-3115-5

Christensen, G. J. M., and Brüggemann, H. (2014). Bacterial skin commensals and their role as host guardians. Beneficial Microbes 5, 201-215. doi: 10.3920/BM2012.0062

Christensen, G. J. M., Scholz, C. F. P., Enghild, J., Rohde, H., Kilian, M., Thürmer, A., et al. (2016). Antagonism between Staphylococcus epidermidis and Propionibacterium acnes and its genomic basis. BMC Genomics 17:5. doi: 10.1186/s12864-016-2489-5

Christie, K., Atkins, N., and Munch-Petersen, E. A. (1944). Note on a lytic phenomenon shown by group B Streptococci. Aust. J. Exp. Biol Med. 22, 197-200. doi: 10.1038/icb.1944.26

Claesen, J., Spagnolo, J. B., Ramos, S. F., Kurita, K. L., Byrd, A. L., Aksenov, A. A., et al. (2020). A Cutibacterium acnes antibiotic modulates human skin microbiota composition in hair follicles. Sci. Transl. Med. 12:5445. doi: 10.1126/scitranslmed.aay5445

Clayton, J. J., Baig, W., Reynolds, G. W., and Sandoe, J. A. T. (2006). Endocarditis caused by Propionibacterium species: a report of three cases and a review of clinical features and diagnostic difficulties. J. Med. Microbiol. 55, 981-987. doi: 10.1099/jmm.0.46613-0

Coenye, T., Peeters, E., and Nelis, H. J. (2007). Biofilm formation by Propionibacterium acnes is associated with increased resistance to antimicrobial agents and increased production of putative virulence factors. Res. Microbiol. 158, 386-392. doi: 10.1016/j.resmic.2007.02.001

Cogen, A. L., Nizet, V., and Gallo, R. L. (2008). Skin microbiota: a source of disease or defence? Br. J. Dermatol. 158, 442-455. doi: 10.1111/j.1365-2133.2008.08437.x

Cohen, R. J., Shannon, B. A., McNeal, J. E., Shannon, T., and Garrett, K. L. (2005). Propionibacterium acnes associated with inflammation in radical prostatectomy specimens: a possible link to cancer evolution? J. Urol. 173, 1969-1974. doi: 10.1097/01.ju.0000158161.15277.78

Conen, A., Walti, L. N., Merlo, A., Fluckiger, U., Battegay, M., and Trampuz, A. (2008). Characteristics and treatment outcome of cerebrospinal fluid shuntassociated infections in adults: a retrospective analysis over an 11-year period. Clin. Infect. Dis. 47, 73-82. doi: 10.1086/588298

Conly, J. M., and Stein, K. (1992). The production of menaquinones (vitamin K2) by intestinal bacteria and their role in maintaining coagulation homeostasis. Progr. Food Nutr. Sci. 16, 307-343.

da Silva, J. B. N. F., de Oliveira, S. K. M., Campos, I. A., de CarvalhoJúnior, C. H. R., Coutinho, T. da C., Silva, T. G. (2013). Propionibacterium acnes-killed attenuates the inflammatory response and protects mice from sepsis by modulating inflammatory factors. Brazil. J. Infect. Dis. 17, 20-26. doi: 10.1016/j.bjid.2012.08.023 
Dagnelie, M. A., Corvec, S., Saint-Jean, M., Bourdès, V., Nguyen, J. M., Khammari, A., et al. (2018). Decrease in diversity of Propionibacterium acnes phylotypes in patients with severe acne on the back. Acta Derm. Venereol. 98, 262-267. doi: 10.2340/00015555-2847

Dagnelie, M. A., Corvec, S., Saint-Jean, M., Nguyen, J. M., Khammari, A., and Dréno, B. (2019). Cutibacterium acnes phylotypes diversity loss: a trigger for skin inflammatory process. J. Eur. Acad. Dermatol. Venereol. 33, 2340-2348. doi: $10.1111 /$ jdv.15795

Davidsson, S., Carlsson, J., Mölling, P., Gashi, N., Andrén, O., Andersson, S. O., et al. (2017). Prevalence of Flp Pili-encoding plasmids in Cutibacterium acnes isolates obtained from prostatic tissue. Front. Microbiol. 8:2241. doi: 10.3389/fmicb.2017.02241

Davidsson, S., Mölling, P., Rider, J. R., Unemo, M., Karlsson, M. G., Carlsson, J., et al. (2016). Frequency and typing of Propionibacterium acnes in prostate tissue obtained from men with and without prostate cancer. Infect. Agents Cancer 11:7. doi: 10.1186/s13027-016-0084-7

Dekio, I., McDowell, A., Sakamoto, M., Tomida, S., and Ohkuma, M. (2019). Proposal of new combination, Cutibacterium acnes subsp. Elongatum comb. nov., and emended descriptions of the genus cutibacterium, Cutibacterium acnes subsp. acnes and Cutibacterium acnes subsp. defendens. Int. J. Syst. Evol. Microbiol. 69, 1087-1092. doi: 10.1099/ijsem.0.003274

del Pozo, J. L., Tran, N. v., Petty, P. M., Johnson, C. H., Walsh, M. F., Bite, U., et al. (2009). Pilot study of association of bacteria on breast implants with capsular contracture. J. Clin. Microbiol. 47, 1333-1337. doi: 10.1128/JCM.00096-09

Delgado, S., Suarez, A., and Mayo, B. (2011). Identification, typing and characterisation of Propionibacterium strains from healthy mucosa of the human stomach. Int. J. Food Microbiol. 149, 65-72. doi: 10.1016/j.ijfoodmicro.2011.01.028

Dessinioti, C., and Katsambas, A. (2017). Propionibacterium acnes and antimicrobial resistance in acne. Clin. Dermatol. 35, 163-167. doi: 10.1016/j.clindermatol.2016.10.008

Disis, M. L. (2010). Immune regulation of cancer. J. Clin. Oncol. 28, 4531-4538. doi: 10.1200/JCO.2009.27.2146

Dodson, C. C., Craig, E. v., Cordasco, F. A., Dines, D. M., Dines, J. S., DiCarlo, E., et al. (2010). Propionibacterium acnes infection after shoulder arthroplasty: a diagnostic challenge. J. Shoulder Elbow Surg. 19, 303-307. doi: 10.1016/j.jse.2009.07.065

Dréno, B., Dagnelie, M. A., Khammari, A., and Corvec, S. (2020). The skin microbiome: a new actor in inflammatory acne. Am. J. Clin. Dermatol. 21, 18-24. doi: 10.1007/s40257-020-00531-1

Dréno, B., Pécastaings, S., Corvec, S., Veraldi, S., Khammari, A., and Roques, C. (2018). Cutibacterium acnes (Propionibacterium acnes) and acne vulgaris: a brief look at the latest updates. J. Eur. Acad. Dermatol. Venereol. 32, 5-14. doi: $10.1111 /$ jdv.15043

Eady, E. A., Gloor, M., and Leyden, J. J. (2003). Propionibacterium acnes resistance: a worldwide problem. Dermatology 206, 54-56. doi: 10.1159/000067822

Eisenhofer, R., Minich, J. J., Marotz, C., Cooper, A., Knight, R., and Weyrich, L. S. (2019). Contamination in low microbial biomass microbiome studies: issues and recommendations. Trends Microbiol. 27, 105-117. doi: 10.1016/j.tim.2018.11.003

Eishi, Y. (2013). Etiologic link between sarcoidosis and Propionibacterium acnes. Respir Investig. 51, 56-68. doi: 10.1016/j.resinv.2013.01.001

el Sayed, F., Roux, A. L., Sapriel, G., Salomon, E., Bauer, T., Gaillard, J. L., et al. (2019). Molecular typing of multiple isolates is essential to diagnose Cutibacterium acnes orthopedic device-related infection. Clin. Infect. Dis. 68, 1942-1945. doi: 10.1093/cid/ciy952

Erdei, L., Bolla, B. S., Bozó, R., Tax, G., Urbán, E., Burián, K., et al. (2021). Tumour necrosis factor alpha-induced protein 3 negatively regulates Cutibacterium acnes-induced innate immune events in epidermal keratinocytes. Acta Derm Venereol. 101:adv00369. doi: 10.2340/00015555-3707

Erdei, L., Bolla, B. S., Bozó, R., Tax, G., Urbán, E., Kemény, L., et al. (2018). TNIP1 regulates Cutibacterium acnes-induced innate immune functions in epidermal keratinocytes. Front. Immunol. 9:2155. doi: 10.3389/fimmu.2018.02155

Ertürk Bergdahl, G., Andersson, T., Allhorn, M., Yngman, S., Timm, R., and Lood, R. (2019). In vivo detection and absolute quantification of a secreted bacterial factor from skin using molecularly imprinted polymers in a surface plasmon resonance biosensor for improved diagnostic abilities. ACS Sensors 4, 717-725. doi: 10.1021 /acssensors.8b01642
Ertürk, G., Hedström, M., Mattiasson, B., Ruzgas, T., and Lood, R. (2018). Highly sensitive detection and quantification of the secreted bacterial benevolence factor RoxP using a capacitive biosensor: a possible early detection system for oxidative skin diseases. PLoS ONE 13:193754. doi: 10.1371/journal.pone.0193754

Ertürk, G., and Lood, R. (2018). Ultrasensitive detection of biomarkers by using a molecular imprinting based capacitive biosensor. J. Visual. Exp. 2018:57208. doi: $10.3791 / 57208$

Esteban, J., Alvarez-Alvarez, B., Blanco, A., Fernández-Roblas, R., Gadea, I., Garcia-Cañete, J., et al. (2013). Prolonged incubation time does not increase sensitivity for the diagnosis of implant-related infection using samples prepared by sonication of the implants. Bone Joint J. 95-B, 1001-1006. doi: 10.1302/0301-620X.95B7.31174

Esteban, J., Gomez-Barrena, E., Cordero, J., Martín-de-Hijas, N. Z., Kinnari, T. J., and Fernandez-Roblas, R. (2008). Evaluation of quantitative analysis of cultures from sonicated retrieved orthopedic implants in diagnosis of orthopedic infection. J. Clin. Microbiol. 46, 488-492. doi: 10.1128/JCM.01762-07

Evans, C. A., Smith, W. M., Johnston, E. A., and Giblett, E. R. (1950). Bacterial flora of the normal human skin. J. Invest. Dermatol. 15, 305-324. doi: 10.1038/jid.1950.105

Fassi Fehri, L., Mak, T. N., Laube, B., Brinkmann, V., Ogilvie, L. A., Mollenkopf, H., et al. (2011). Prevalence of Propionibacterium acnes in diseased prostates and its inflammatory and transforming activity on prostate epithelial cells. Int. J. Med. Microbiol. 301, 69-78. doi: 10.1016/j.ijmm.2010.08.014

Figa, R., Muñetón, D., Gómez, L., Matamala, A., Lung, M., Cuchi, E., et al. (2017). Periprosthetic joint infection by Propionibacterium acnes: clinical differences between monomicrobial versus polymicrobial infection. Anaerobe 44, 143-149. doi: 10.1016/j.anaerobe.2017.03.008

Fijan, S. (2014). Microorganisms with claimed probiotic properties: an overview of recent literature. Int. J. Environ. Res. Public Health 11, 4745-4767. doi: 10.3390/ijerph110504745

Fink, B., and Sevelda, F. (2017). Periprosthetic joint infection of shoulder arthroplasties: diagnostic and treatment options. BioMed Res. Int. 2017:4582756. doi: 10.1155/2017/4582756

Fischer, K., Tschismarov, R., Pilz, A., Straubinger, S., Carotta, S., McDowell, A., et al. (2020). Cutibacterium acnes infection induces type I interferon synthesis through the cGAS-STING pathway. Front. Immunol. 11:571334. doi: 10.3389/fimmu.2020.571334

Fitz-Gibbon, S., Tomida, S., Chiu, B. H., Nguyen, L., Du, C., Liu, M., et al. (2013). Propionibacterium acnes strain populations in the human skin microbiome associated with acne. J. Investig. Dermatol. 133, 2152-2160. doi: 10.1038/jid.2013.21

Francuzik, W., Franke, K., Schumann, R. R., Heine, G., and Worm, M. (2018). Propionibacterium acnes abundance correlates inversely with Staphylococcus aureus: data from atopic dermatitis skin microbiome. Acta Derm. Venereol. 98, 490-495. doi: 10.2340/00015555-2896

Frangiamore, S. J., Saleh, A., Grosso, M. J., Alolabi, B., Bauer, T. W., Iannotti, J. P., et al. (2015). Early versus late culture growth of Propionibacterium acnes in revision shoulder arthroplasty. J. Bone Joint Surg. Am. 97, 1149-1158. doi: 10.2106/JBJS.N.00881

Fujimura, S., and Nakamura, T. (1978). Purification and properties of a bacteriocin-like substance (Acnecin) of oral Propionibacterium acnes. Antimicrob. Agents Chemother. 14, 893-898. doi: 10.1128/AAC.14.6.893

Gannesen, A. v., Zdorovenko, E. L., Botchkova, E. A., Hardouin, J., Massier, S., Kopitsyn, D. S., et al. (2019). Composition of the biofilm matrix of Cutibacterium acnes acneic strain RT5. Front. Microbiol. 10:1284. doi: $10.3389 /$ fmicb.2019.01284

Gao, Z., Tseng, C. H., Strober, B. E., Pei, Z., and Blaser, M. J. (2008). Substantial alterations of the cutaneous bacterial biota in psoriatic lesions. PLoS ONE 3:2719. doi: 10.1371/journal.pone.0002719

Gharamti, A. A., and Kanafani, Z. A. (2017). Cutibacterium (formerly Propionibacterium) acnes infections associated with implantable devices. Expert Rev. Anti. Infect. Ther. 15, 1083-1094. doi: 10.1080/14787210.2017.1404452

Girolamo, S., di Puorger, C., Castiglione, M., Vogel, M., Gébleux, R., Briendl, M., et al. (2019). Characterization of the housekeeping sortase from the human pathogen Propionibacterium acnes: first investigation of a class F sortase. Biochem. J. 476, 665-682. doi: 10.1042/BCJ20180885 
Gollnick, H. P., and Zouboulis, C. C. (2014). Not all acne is acne vulgaris. Dtsch Arztebl Int. 111, 301-312. doi: 10.3238/arztebl.2014.0301

González-Sánchez, E., Corral, M. J., Mohamed Fawzi, E., Rodríguez-Bertos, A., Alunda, J. M., and Cuquerella, M. (2014). LPS and inactivated Propionibacterium acnes elicit a partially protective response in primary infections of Heligmosomoides polygyrus. Vet. Parasitol. 203, 231-236. doi: 10.1016/j.vetpar.2014.02.026

Grange, P. A., Raingeaud, J., Morelle, W., Marcelin, A. G., Calvez, V., and Dupin, N. (2017). Characterization of a Propionibacterium acnes surface protein as a fibrinogen-binding protein. Sci. Rep. 7:3. doi: 10.1038/s41598-017-06940-3

Gribbon, E. M., Cunliffe, W. J., and Holland, K. T. (1993). Interaction of Propionibacterium acnes with skin lipids in vitro. J. Gen. Microbiol. 139, 1745-1751. doi: 10.1099/00221287-139-8-1745

Griffiths, C. E. M., van de Kerkhof, P., and Czarnecka-Operacz, M. (2017). Psoriasis and atopic dermatitis. Dermatol. Therapy 7, 31-41. doi: 10.1007/s13555-016-0167-9

Grosso, M. J., Frangiamore, S. J., Yakubek, G., Bauer, T. W., Iannotti, J. P., and Ricchetti, E. T. (2018). Performance of implant sonication culture for the diagnosis of periprosthetic shoulder infection. J. Shoulder Elbow Surg. 27, 211-216. doi: 10.1016/j.jse.2017.08.008

Hammami, R., Fernandez, B., Lacroix, C., and Fliss, I. (2013). Anti-infective properties of bacteriocins: an update. Cell. Mol. Life Sci. 70, 2947-2967. doi: 10.1007/s00018-012-1202-3

Harmsen, H. J. M., and de Goffau, M. C. (2016). "The human gut microbiota," in Advances in Experimental Medicine and Biology (New York, NY: Springer New York LLC), 95-108. doi: 10.1007/978-3-319-31248-4_7

He, X., Shang, J., Li, F., and Liu, H. (2015). Yeast cell surface display of linoleic acid isomerase from Propionibacterium acnes and its application for the production of trans-10, cis-12 conjugated linoleic acid. Biotechnol. Appl. Biochem. 62, 1-8. doi: $10.1002 /$ bab.1249

Hevia, A., Delgado, S., Sánchez, B., and Margolles, A. (2015). Molecular players involved in the interaction between beneficial bacteria and the immune system. Front. Microbiol. 6:1285. doi: 10.3389/fmicb.2015.01285

Holland, C., Mak, T. N., Zimny-Arndt, U., Schmid, M., Meyer, T. F., Jungblut, P. R., et al. (2010). Proteomic identification of secreted proteins of Propionibacterium acnes. BMC Microbiol. 10:230. doi: 10.1186/1471-2180-10-230

Holmberg, A., Lood, R., Mörgelin, M., Söderquist, B., Holst, E., Collin, M., et al. (2009). Biofilm formation by Propionibacterium acnes is a characteristic of invasive isolates. Clin. Microbiol. Infect. 15, 787-795. doi: 10.1111/j.1469-0691.2009.0 2747.x

Hudek, R., Brobeil, A., Brüggemann, H., Sommer, F., Gattenlöhner, S., and Gohlke, F. (2020). Cutibacterium acnes is an intracellular and intra-articular commensal of the human shoulder joint. J. Shoulder Elbow Surg. 30:42. doi: 10.1016/j.jse.2020.01.042

Hudek, R., Sommer, F., Abdelkawi, A. F., Kerwat, M., Müller, H. H., and Gohlke, F. (2016). Propionibacterium acnes in shoulder surgery: is loss of hair protective for infection? J. Shoulder Elbow Surg. 25, 973-980. doi: 10.1016/j.jse.2015.10.018

Jahns, A. C., and Alexeyev, O. A. (2014). Three dimensional distribution of Propionibacterium acnes biofilms in human skin. Exp. Dermatol. 23, 687-689. doi: $10.1111 /$ exd.12482

Jasson, F., Nagy, I., Knol, A. C., Zuliani, T., Khammari, A., and Dréno, B. (2013). Different strains of Propionibacterium acnes modulate differently the cutaneous innate immunity. Exp. Dermatol. 22, 587-592. doi: 10.1111/exd.12206

Jha, S. C., and Sairyo, K. (2020). The role of Propionibacterium acnes in and modic type 1 changes: a literature review. J. Medical Investig. 67, 21-26. doi: 10.2152/jmi.67.21

Johnson, T., Kang, D., Barnard, E., and Li, H. (2016). Strain-level differences in porphyrin production and regulation in Propionibacterium acnes elucidate disease associations. $m$ Sphere 1:15. doi: 10.1128/mSphere.00023-15

Kalis, C., Gumenscheimer, M., Freudenberg, N., Tchaptchet, S., Fejer, G., Heit, A., et al. (2005). Requirement for TLR9 in the immunomodulatory activity of Propionibacterium acnes. J. Immunol. 174, 4295-4300. doi: 10.4049/jimmunol.174.7.4295

Karadag, A. S., Aslan Kayiran, M., Wu, C. Y., Chen, W., and Parish, L. C. (2021). Antibiotic resistance in acne: changes, consequences and concerns. J. Eur. Acad. Dermatol. Venereol. 35, 73-78. doi: 10.1111/jdv.16686
Karau, M. J., Greenwood-Quaintance, K. E., Schmidt, S. M., Tran, N. v., Convery, P. A., Jacobson, S. R., et al. (2013). Microbial biofilms and breast tissue expanders. BioMed. Res. Int. 2013:254940. doi: 10.1155/2013/254940

Kennedyl, J. D., and Conley, F. K. (1989). Effect of intracerebrally injected Corynebacterium parvum on implanted brain tumor in mice. J. Neurooncol. 7 , 89-101. doi: 10.1007/BF00149383

Khalil, J. G., Gandhi, S. D., Park, D. K., and Fischgrund, J. S. (2019). Cutibacterium acnes in spine pathology: pathophysiology, diagnosis, and management. J. Am. Acad. Orthopaed. Surgeons 27, e633-e640. doi: 10.5435/JAAOS-D-17-00698

Kim, J., Ochoa, M.-T., Krutzik, S. R., Takeuchi, O., Uematsu, S., Legaspi, A. J., et al. (2002). Activation of toll-like receptor 2 in acne triggers inflammatory cytokine responses. J. Immunol. 169, 1535-1541. doi: 10.4049/jimmunol.169.3.1535

Kistowska, M., Gehrke, S., Jankovic, D., Kerl, K., Fettelschoss, A., Feldmeyer, L., et al. (2014). IL-1 $\beta$ drives inflammatory responses to propionibacterium acnes in vitro and in vivo. J. Investig. Dermatol. 134, 677-685. doi: $10.1038 /$ jid.2013.438

Kistowska, M., Meier, B., Proust, T., Feldmeyer, L., Cozzio, A., Kuendig, T., et al. (2015). Propionibacterium acnes promotes Th17 and Th17/Th1 responses in acne patients. J. Investig. Dermatol. 135, 110-118. doi: 10.1038/jid.2014.290

Kitagawa, H., Yamanaka, K., Kakeda, M., Inada, H., Imai, Y., Gabazza, E. C., et al. (2011). Propionibacterium acnes vaccination induces regulatory T cells and Th1 immune responses and improves mouse atopic dermatitis. Exp. Dermatol. 20, 157-158. doi: 10.1111/j.1600-0625.2010.01180.x

Koutsioulis, D., Landry, D., and Guthrie, E. P. (2008). Novel endo-alpha-Nacetylgalactosaminidases with broader substrate specificity. Glycobiology 18, 799-805. doi: 10.1093/glycob/cwn069

Kowalski, T. J., Berbari, E. F., Huddleston, P. M., Steckelberg, J. M., Mandrekar, J. N., and Osmon, D. R. (2007). The management and outcome of spinal implant infections: contemporary retrospective cohort study. Clin. Infect. Dis. 44, 913-920. doi: 10.1086/512194

Kuehnast, T., Cakar, F., Weinhäupl, T., Pilz, A., Selak, S., Schmidt, M. A., et al. (2018). Comparative analyses of biofilm formation among different Cutibacterium acnes isolates. Int. J. Med. Microbiol. 308, 1027-1035. doi: 10.1016/j.ijmm.2018.09.005

Kvich, L., Jensen, P., Justesen, U. S., and Bjarnsholt, T. (2016). Incidence of Propionibacterium acnes in initially culture-negative thioglycollate brothsa prospective cohort study at a Danish University Hospital. Clin. Microbiol. Infect. 22, 941-945. doi: 10.1016/j.cmi.2016.07.036

Lang, S., and Palmer, M. (2003). Characterization of Streptococcus agalactiae CAMP factor as a pore-forming toxin. J. Biol. Chem. 278, 38167-38173. doi: 10.1074/jbc.M303544200

Lavergne, V., Malo, M., Gaudelli, C., Laprade, M., Leduc, S., Laflamme, P., et al. (2017). Clinical impact of positive Propionibacterium acnes cultures in orthopedic surgery. Orthopaed. Traumatol. Surg. Res. 103, 307-314. doi: 10.1016/j.otsr.2016.12.005

LeBlanc, J. G., Chain, F., Martín, R., Bermúdez-Humarán, L. G., Courau, S., and Langella, P. (2017). Beneficial effects on host energy metabolism of shortchain fatty acids and vitamins produced by commensal and probiotic bacteria. Microbial Cell Factories 16:691. doi: 10.1186/s12934-017-0691-z

Lee, M., Ponraja, G., McLeod, K., and Chong, S. (2020). Breast implant illness: a biofilm hypothesis. Plastic Reconstruct. Surg. Glob. Open 8:2994. doi: 10.1097/GOX.0000000000002994

Leyden, J. J., McGinley, K. J., and Vowels, B. (1998). Propionibacterium acnes colonization in acne and nonacne. Dermatology 55-58. doi: 10.1159/000017868

Lheure, C., Grange, P. A., Ollagnier, G., Morand, P., Désiré, N., Sayon, S., et al. (2016). TLR-2 recognizes Propionibacterium acnes CAMP factor 1 from highly inflammatory strains. PLoS ONE 11:167237. doi: 10.1371/journal.pone.0167237

Li, L., Sun, C., Yang, F., Yang, S., Feng, X., Gu, J., et al. (2013). Identification of proteins of Propionibacterium acnes for use as vaccine candidates to prevent infection by the pig pathogen Actinobacillus pleuropneumoniae. Vaccine 31, 5269-5275. doi: 10.1016/j.vaccine.2013.08.054

Liew-Littorin, C., Brüggemann, H., Davidsson, S., Nilsdotter-Augustinsson, H.ellmark, B., and Söderquist, B. (2019). Clonal diversity of Cutibacterium acnes (formerly Propionibacterium acnes) in prosthetic joint infections. Anaerobe 59, 54-60. doi: 10.1016/j.anaerobe.2019.04.011

Lin, Y., Jiao, Y., Yuan, Y., Zhou, Z., Zheng, Y., Xiao, J., et al. (2018). Propionibacterium acnes induces intervertebral disc degeneration by promoting nucleus pulposus cell apoptosis via the 
TLR2/JNK/mitochondrial-mediated pathway article. Emerg. Microb. Infect. 7:2. doi: 10.1038/s41426-017-0002-0

Lin, Z. X., Steed, L. L., Marculescu, C. E., Slone, H. S., and Woolf, S. K. (2020). Cutibacterium acnes infection in orthopedics: microbiology, clinical findings, diagnostic strategies, and management. Orthopedics 43, 52-61. doi: 10.3928/01477447-20191213-02

Lodes, M. J., Secrist, H., Benson, D. R., Jen, S., Shanebeck, K. D., Guderian, J., et al. (2006). Variable expression of immunoreactive surface proteins of Propionibacterium acnes. Microbiology 152, 3667-3681. doi: 10.1099/mic.0.29219-0

Lomholt, H. B., and Kilian, M. (2010). Population genetic analysis of Propionibacterium acnes identifies a subpopulation and epidemic clones associated with acne. PLoS ONE 5:12277. doi: 10.1371/journal.pone.0012277

Lomholt, H. B., and Kilian, M. (2014). Clonality and anatomic distribution on the skin of antibiotic resistant and sensitive Propionibacterium acnes. Acta Derm Venereol. 94, 534-538. doi: 10.2340/00015555-1794

Lomholt, H. B., Scholz, C. F. P., Brüggemann, H., Tettelin, H., and Kilian, M. (2017). A comparative study of Cutibacterium (Propionibacterium) acnes clones from acne patients and healthy controls. Anaerobe 47, 57-63. doi: 10.1016/j.anaerobe.2017.04.006

Mak, T. N., Schmid, M., Brzuszkiewicz, E., Zeng, G., Meyer, R., Sfanos, K. S., et al. (2013). Comparative genomics reveals distinct host-interacting traits of three major human-associated propionibacteria. BMC Genom. 14:640. doi: 10.1186/1471-2164-14-640

Manniche, C., and O'Neill, S. (2019). New insights link low-virulent disc infections to the etiology of severe disc degeneration and Modic changes. Fut. Sci. 5:FSO389. doi: 10.4155/fsoa-2019-0022

Mayslich, C., Grange, P. A., and Dupin, N. (2021). Cutibacterium acnes as an opportunistic pathogen: an update of its virulence-associated factors. Microorganisms 9:303. doi: 10.3390/microorganisms9020303

Mazmanian, S. K., Round, J. L., and Kasper, D. L. (2008). A microbial symbiosis factor prevents intestinal inflammatory disease. Nature 453, 620-625. doi: $10.1038 /$ nature 07008

McDowell, A., Barnard, E., Nagy, I., Gao, A., Tomida, S., Li, H., et al. (2012). An expanded multilocus sequence typing scheme for propionibacterium acnes: investigation of "pathogenic", "commensal" and antibiotic resistant strains. PLoS ONE 7:41480. doi: 10.1371/journal.pone.0041480

McDowell, A., Gao, A., Barnard, E., Fink, C., Murray, P. I., Dowson, C. G., et al. (2011). A novel multilocus sequence typing scheme for the opportunistic pathogen Propionibacterium acnes and characterization of type I cell surfaceassociated antigens. Microbiology 157, 1990-2003. doi: 10.1099/mic.0.049676-0

McDowell, A., McLaughlin, J., and Layton, A. M. (2020). Is Cutibacterium (previously Propionibacterium) acnes a potential pathogenic factor in the aetiology of the skin disease progressive macular hypomelanosis? J. Eur. Acad. Dermatol. Venereol. 35, 338-344. doi: 10.1111/jdv.16789

McDowell, A., Nagy, I., Magyari, M., Barnard, E., and Patrick, S. (2013). The opportunistic pathogen Propionibacterium acnes: insights into typing, human disease, clonal diversification and CAMP factor evolution. PLoS ONE 8:70897. doi: 10.1371/journal.pone.0070897

McLaughlin, J., Watterson, S., Layton, A. M., Bjourson, A. J., Barnard, E., and McDowell, A. (2019). Propionibacterium acnes and acne vulgaris: new insights from the integration of population genetic, multiomic, biochemical and host-microbe studies. Microorganisms 7:50128. doi: 10.3390/microorganisms7050128

Megyeri, K., Orosz, L., Bolla, S., Erdei, L., Rázga, Z., Seprényi, G., et al. (2018). Propionibacterium acnes induces autophagy in keratinocytes: involvement of multiple mechanisms. J. Investig. Dermatol. 138, 750-759. doi: 10.1016/j.jid.2017.11.018

Miskin, J. E., Farrell, A. M., Cunliffe, W. J., and Holland, K. T. (1997). Propionibacterium acnes, a resident of lipid-rich human skin, produces a $33 \mathrm{kDa}$ extracellular lipase encoded by gehA. Microbiology 143, 1745-1755. doi: 10.1099/00221287-143-5-1745

Mollerup, S., Friis-Nielsen, J., Vinner, L., Hansen, T. A., Richter, S. R., Fridholm, H., et al. (2016). Propionibacterium acnes: disease-causing agent or common contaminant? detection in diverse patient samples by next- generation sequencing. J. Clin. Microbiol. 54, 980-987. doi: 10.1128/JCM.02723-15
Moore, W. E., and Cato, E. P. (1963). Validity of Propionibacterium acnes (Gilchrist) Douglas and Gunter comb. nov. J. Bacteriol. 85, 870-874. doi: 10.1128/JB.85.4.870-874.1963

Morgenstern, C., Renz, N., Cabric, S., Perka, C., and Trampuz, A. (2018). Multiplex polymerase chain reaction and microcalorimetry in synovial fluid: can pathogen-based detection assays improve the diagnosis of septic arthritis? J. Rheumatol. 45, 1588-1593. doi: 10.3899/jrheum.180311

Morrison, D. J., and Preston, T. (2016). Formation of short chain fatty acids by the gut microbiota and their impact on human metabolism. Gut Microbes 7 , 189-200. doi: 10.1080/19490976.2015.1134082

Nagata, K., Eishi, Y., Uchida, K., Yoneda, K., Hatanaka, H., and Yasuhara, T., et al (2017). Immunohistochemical detection of Propionibacterium acnes in the retinal granulomas in patients with ocular sarcoidosis. Sci. Rep. 7:15226. doi: 10.1038/s41598-017-15710-0

Nagy, I., Pivarcsi, A., Koreck, A., Széll, M., Urbán, E., and Kemény, L. (2005). Distinct strains of Propionibacterium acnes induce selective human $\beta$-defensin- 2 and interleukin-8 expression in human keratinocytes through toll-like receptors. J. Investig. Dermatol. 124, 931-938. doi: 10.1111/j.0022-202X.2005.23705.X

Naik, S., Bouladoux, N., Linehan, J. L., Han, S. J., Harrison, O. J., Wilhelm, C., et al. (2015). Commensal-dendritic-cell interaction specifies a unique protective skin immune signature. Nature 520, 104-108. doi: 10.1038/nature14052

Nakamura, K., O’Neill, A. M., Williams, M. R., Cau, L., Nakatsuji, T., Horswill, A. R., et al. (2020). Short chain fatty acids produced by Cutibacterium acnes inhibit biofilm formation by Staphylococcus epidermidis. Sci. Rep. 10:9. doi: 10.1038/s41598-020-77790-9

Nakamura, T., Fujimura, S., Obata, N., Yamazaki, N., and Kanagawa, N. (1978). Bacteriocin (acnecin) activity of oral Propionibacterium acnes. Bulletin Tokyo Dental College 19, 235-244.

Nakamura, T., Furukawa, A., Uchida, K., Ogawa, T., Tamura, T., Sakonishi, D., et al. (2016). Autophagy induced by intracellular infection of Propionibacterium acnes. PLoS ONE 11:156298. doi: 10.1371/journal.pone.0156298

Nakase, K., Aoki, S., Sei, S., Fukumoto, S., Horiuchi, Y., Yasuda, T., et al. (2020). Characterization of acne patients carrying clindamycin-resistant Cutibacterium acnes: a Japanese multicenter study. J. Dermatol. 47, 863-869. doi: $10.1111 / 1346-8138.15397$

Nakase, K., Hayashi, N., Akiyama, Y., Aoki, S., and Noguchi, N. (2017). Antimicrobial susceptibility and phylogenetic analysis of Propionibacterium acnes isolated from acne patients in Japan between 2013 and 2015. J. Dermatol. 44, 1248-1254. doi: 10.1111/1346-8138.13913

Nakase, K., Okamoto, Y., Aoki, S., and Noguchi, N. (2018). Longterm administration of oral macrolides for acne treatment increases macrolide-resistant Propionibacterium acnes. J. Dermatol. 45, 340-343. doi: 10.1111/1346-8138.14178

Nakatsuji, T., Liu, Y. T., Huang, C. P., Zouboulis, C. C., Gallo, R. L., and Huang, C. M. (2008). Vaccination targeting a surface sialidase of $P$. acnes: implication for new treatment of acne vulgaris. PLoS ONE 3:e1551. doi: 10.1371/journal.pone.0001551

Nakatsuji, T., Tang, D., Chu, C., Zhang, L., Gallo, R. L., and Huang, C. M. (2011). Propionibacterium acnes camp factor and host acid sphingomyelinase contribute to bacterial virulence: potential targets for inflammatory acne treatment. PLoS ONE 6:14797. doi: 10.1371/journal.pone.0014797

Namdari, S., Nicholson, T., and Parvizi, J. (2020). Cutibacterium acnes is isolated from air swabs: time to doubt the value of traditional cultures in shoulder surgery? Archiv. Bone Joint Surg. 8, 506-510. doi: 10.22038/abjs.2020.40642.2095

Narendhirakannan, R. T., and Hannah, M. A. C. (2013). Oxidative stress and skin cancer: an overview. Ind. J. Clin. Biochem. 28, 110-115. doi: 10.1007/s12291-012-0278-8

Nazipi, S., Stødkilde, K., Scavenius, C., and Brüggemann, H. (2017). The skin bacterium Propionibacterium acnes employs two variants of hyaluronate lyase with distinct properties. Microorganisms 5:57. doi: 10.3390/microorganisms5030057

Nisbet, M., Briggs, S., Ellis-Pegler, R., Thomas, M., and Holland, D. (2007). Propionibacterium acnes: an under-appreciated cause of postneurosurgical infection. J. Antimicrob. Chemotherapy 60, 1097-1103. doi: $10.1093 / \mathrm{jac} / \mathrm{dkm} 351$ 
Nodzo, S. R., Boyle, K. K., Bhimani, S., Duquin, T. R., Miller, A. O., and Westrich, G. H. (2017). Propionibacterium acnes host inflammatory response during periprosthetic infection is joint specific. HSS J. 13, 159-164. doi: 10.1007/s11420-016-9528-2

Ohrt-Nissen, S., Fritz, B. G., Walbom, J., Kragh, K. N., Bjarnsholt, T., Dahl, B., et al. (2018). Bacterial biofilms: a possible mechanism for chronic infection in patients with lumbar disc herniation - a prospective proof-ofconcept study using fluorescence in situ hybridization. APMIS 126, 440-447. doi: 10.1111/apm.12841

Okuda, K. I., Nagahori, R., Yamada, S., Sugimoto, S., Sato, C., Sato, M., et al. (2018). The composition and structure of biofilms developed by Propionibacterium acnes isolated from cardiac pacemaker devices. Front. Microbiol. 9:182. doi: $10.3389 /$ fmicb. 2018.00182

Paillot, R. (2013). A systematic review of the immune-modulators Parapoxvirus ovis and Propionibacterium acnes for the prevention of respiratory disease and other infections in the horse. Vet. Immunol. Immunopathol. 153, 1-9. doi: 10.1016/j.vetimm.2013.01.010

Peters, B. A., and Hay, C. A. (1990). Adjunct treatment of equine respiratory disease complex (ERDC) with the propionibacterium acnes, immunostimulant, EqStim ${ }^{\circledR}$. J. Equine Vet. Sci. 10, 399-400. doi: 10.1016/S0737-0806(06)80132-2

Petersen, R. L. W., Scholz, C. F. P., Jensen, A., Brüggemann, H., and Lomholt, H. B. (2017). Propionibacterium acnes phylogenetic type III is associated with progressive macular hypomelanosis. Eur. J. Microbiol. Immunol. 7, 37-45. doi: $10.1556 / 1886.2016 .00040$

Petersson, F., Kilsgård, O., Shannon, O., and Lood, R. (2018). Platelet activation and aggregation by the opportunistic pathogen Cutibacterium (Propionibacterium) acnes. PLOS ONE 13:192051. doi: 10.1371/journal.pone.0192051

Pickering, A. C., Vitry, P., Prystopiuk, V., Garcia, B., Höök, M., Schoenebeck, J., et al. (2019). Host-specialized fibrinogen-binding by a bacterial surface protein promotes biofilm formation and innate immune evasion. PLoS Pathog. 15:7816. doi: 10.1371/journal.ppat.1007816

Piggott, D. A., Higgins, Y. M., Melia, M. T., Ellis, B., Carroll, K. C., McFarland, E. G., et al. (2015). Characteristics and treatment outcomes of Propionibacterium acnes prosthetic shoulder infections in adults. Open Forum Infect. Dis. 3:ofv191. doi: 10.1093/ofid/ofv191

Platsidaki, E., and Dessinioti, C. (2018). Recent advances in understanding Propionibacterium acnes (Cutibacterium acnes) in acne [version 1; referees: 2 approved]. F1000Research 7:15659. doi: 10.12688/f1000research.15659.1

Portillo, M. E., Corvec, S., Borens, O., and Trampuz, A. (2013). Propionibacterium acnes: an underestimated pathogen in implant-associated infections. BioMed Res. Int. 2013:804391. doi: 10.1155/2013/804391

Qin, M., Pirouz, A., Kim, M. H., Krutzik, S. R., Garbán, H. J., and Kim, J. (2014). Propionibacterium acnes induces IL-1 $\beta$ secretion via the NLRP3 inflammasome in human monocytes. J. Investig. Dermatol. 134, 381-388. doi: 10.1038/jid.2013.309

Reis, V. O., Silva, J. C., Souza, G. T., Semedo, P., Buscariollo, B., Pereira, R. L., et al. (2012). The polysaccharide fraction of Propionibacterium acnes modulates the development of experimental focal segmental glomerulosclerosis. Immunobiology 217, 831-841. doi: 10.1016/j.imbio.2011.12.003

Reischies, F. M. J., Krause, R., Holzer, J., Tiefenbacher, F., Winter, R., Eylert, G., et al. (2017). What can we learn from sonication results of breast implants? PLoS ONE 12:e0182267. doi: 10.1371/journal.pone.0182267

Renz, N., Mudrovcic, S., Perka, C., and Trampuz, A. (2018). Orthopedic implantassociated infections caused by Cutibacterium spp. - a remaining diagnostic challenge. PLoS ONE 13:202639. doi: 10.1371/journal.pone.0202639

Rolfe, R. D., Hentges, D. J., Campbell, B. J., and Barrett, J. T. (1978). Factors related to the oxygen tolerance of anaerobic bacteria. Appl. Environ. Microbiol. 36, 306-313. doi: 10.1128/AEM.36.2.306-313.1978

Ross, J. I., Eady, E. A., Carnegie, E., and Cove, J. H. (2002). Detection of transposon Tn5432-mediated macrolide-lincosamide-streptogramin B (MLSB) resistance in Cutaneous propionibacteria from six European cities. J. Antimicrob. Chemother. 49, 165-168. doi: 10.1093/jac/49.1.165

Salar-Vidal, L., Martin-Garcia, M., Auñón, A., and Esteban, J. (2020). Cutibacterium spp. isolated from orthopaedic implant-associated infection: a not-so-slowly growing organism. Enfermedades Infecciosas Microbiologia Clinica. S0213, 30223-30228. doi: 10.1016/j.eimc.2020.05.024
Sanford, J. A., O’Neill, A. M., Zouboulis, C. C., and Gallo, R. L. (2019). Short-chain fatty acids from Cutibacterium acnes activate both a canonical and epigenetic inflammatory response in human sebocytes. J. Immunol. 202, 1767-1776. doi: 10.4049/jimmunol.1800893

Sanford, J. A., Zhang, L. J., Williams, M. R., Gangoiti, J. A., Huang, C. M., and Gallo, R. L. (2016). Inhibition of HDAC8 and HDAC9 by microbial shortchain fatty acids breaks immune tolerance of the epidermis to TLR ligands. Sci. Immunol. 1:aah4609. doi: 10.1126/sciimmunol.aah4609

Sasaki, N., Sakai, K., and Takazoe, I. (1980). An improved medium for the selective isolation of Propionibacterium acnes from the human oral cavity and skin. J. Dent. Res. 59, 1518-1519. doi: 10.1177/00220345800590091301

Schaller, M., Loewenstein, M., Borelli, C., Jacob, K., Vogeser, M., Burgdorf, W. H. C., et al. (2005). Induction of a chemoattractive proinflammatory cytokine response after stimulation of keratinocytes with Propionibacterium acnes and coproporphyrin III. Br. J. Dermatol. 153, 66-71. doi: 10.1111/j.1365-2133.2005.06530.x

Scholz, C. F. P., Brüggemann, H., Lomholt, H. B., Tettelin, H., and Kilian, M. (2016). Genome stability of Propionibacterium acnes: A comprehensive study of indels and homopolymeric tracts. Sci. Rep. 6:srep20662. doi: 10.1038/srep20662

Scholz, C. F. P., Jensen, A., Lomholt, H. B., Brüggemann, H., and Kilian, M (2014). A novel high-resolution single locus sequence typing scheme for mixed populations of Propionibacterium acnes in vivo. PLOS ONE 9:104199. doi: 10.1371/journal.pone.0104199

Scholz, C. F. P., and Kilian, M. (2016). The natural history of cutaneous propionibacteria, and reclassification of selected species within the genus Propionibacterium to the proposed novel genera Acidipropionibacterium gen. nov., Cutibacterium gen. nov. and Pseudopropionibacterium gen. nov. Int. J. Syst. Evol. Microbiol. 66, 4422-4432. doi: 10.1099/ijsem.0.001367

Shah, N. B., Tande, A. J., Patel, R., and Berbari, E. F. (2015). Anaerobic prosthetic joint infection. Anaerobe 36, 1-8. doi: 10.1016/j.anaerobe.2015.08.003

Shannon, B. A., Cohen, R. J., and Garrett, K. L. (2006). Polymerase chain reactionbased identification of Propionibacterium acnes types isolated from the male urinary tract: evaluation of adolescents, normal adults and men with prostatic pathology. BJU Int. 98, 388-392. doi: 10.1111/j.1464-410X.2006.06273.x

Sheffer-Levi, S., Rimon, A., Lerer, V., Shlomov, T., Coppenhagen-Glazer, S., Rakov, C., et al. (2020). Antibiotic susceptibility of Cutibacterium acnes strains isolated from Israeli Acne Patients. Acta Derm. Venereol. 100:adv00295. doi: 10.2340/00015555-3654

Shields, M. v., Abdullah, L., and Namdari, S. (2016). The challenge of Propionibacterium acnes and revision shoulder arthroplasty: a review of current diagnostic options. J. Shoulder Elbow Surg. 25, 1034-1040. doi: 10.1016/j.jse.2016.01.009

Shinohara, D. B., Vaghasia, A. M., Yu, S.-H., Mak, T. N., Brüggemann, H., Nelson, W. G., et al. (2013). A mouse model of chronic prostatic inflammation using a human prostate cancer-derived isolate of Propionibacterium acnes. Prostate 73, 1007-1015. doi: 10.1002/pros.22648

Shiono, Y., Ishii, K., Nagai, S., Kakinuma, H., Sasaki, A., Funao, H., et al. (2016). Delayed Propionibacterium acnes surgical site infections occur only in the presence of an implant. Sci. Rep. 6:srep32758. doi: 10.1038/srep32758

Shrestha, E., White, J. R., Yu, S. H., Kulac, I., Ertunc, O., de Marzo, A. M., et al. (2018). Profiling the urinary microbiome in men with positive versus negative biopsies for prostate cancer. J. Urol. 199, 161-171. doi: 10.1016/j.juro.2017.08.001

Shu, M., Kuo, S., Wang, Y., Jiang, Y., Liu, Y.-T., Gallo, R. L., et al. (2013a). Porphyrin metabolisms in human skin commensal Propionibacterium acnes bacteria: potential application to monitor human radiation risk. Curr. Med. Chem. 20, 562-568. doi: 10.2174/092986713804910085

Shu, M., Wang, Y., Yu, J., Kuo, S., Coda, A., Jiang, Y., et al. (2013b). Fermentation of Propionibacterium acnes, a commensal bacterium in the human skin microbiome, as skin probiotics against methicillin-resistant Staphylococcus aureus. PLoS ONE 8:55380. doi: 10.1371/journal.pone.0055380

Sigmund, I. K., Windhager, R., Sevelda, F., Staats, K., Puchner, S. E., Stenicka, S., et al. (2019). Multiplex PCR Unyvero i60 ITI application improves detection of low-virulent microorganisms in periprosthetic joint infections. Int. Orthop. 43, 1891-1898. doi: 10.1007/s00264-018-4136-z

Silva, M. J. B., Carneiro, M. B. H., dos Anjos Pultz, B., Pereira Silva, D., Lopes, M. E. D. M., and dos Santos, L. M. (2015). The multifaceted role of commensal 
microbiota in homeostasis and gastrointestinal diseases. J. Immunol. Res. 2015:321241. doi: 10.1155/2015/321241

Sohail, M. R., Gray, A. L., Baddour, L. M., Tleyjeh, I. M., and Virk, A. (2009). Infective endocarditis due to Propionibacterium species. Clin. Microbiol. Infect. 15, 387-394. doi: 10.1111/j.1469-0691.2009. 02703.x

Song, J., Zhao, M., Li, Q., Lu, L., Zhou, Y., Zhang, Y., et al. (2019). IL17A can promote Propionibacterium acnes-induced sarcoidosis-like granulomatosis in mice. Front. Immunol. 10:1923. doi: 10.3389/fimmu.2019. 01923

Sörensen, M., Mak, T. N., Hurwitz, R., Ogilvie, L. A., Mollenkopf, H. J., Meyer, T. F., et al. (2010). Mutagenesis of Propionibacterium acnes and analysis of two CAMP factor knock-out mutants. J. Microbiol. Methods 83, 211-216. doi: 10.1016/j.mimet.2010.09.008

Spittaels, K. J., and Coenye, T. (2018). Developing an in vitro artificial sebum model to study Propionibacterium acnes biofilms. Anaerobe 49, 21-29. doi: 10.1016/j.anaerobe.2017.11.002

Stirling, A., Worthington, T., Rafiq, M., Lambert, P. A., and Elliott, T. S. J. (2001). Association between sciatica and Propionibacterium acnes. Lancet 357, 2024-2025. doi: 10.1016/S0140-6736(00) 05109-6

Su, Q., Grabowski, M., and Weindl, G. (2017). Recognition of Propionibacterium acnes by human TLR2 heterodimers. Int. J. Med. Microbiol. 307, 108-112. doi: 10.1016/j.ijmm.2016.12.002

Suzuki, K., Saito, T., Sakai, K., Miyagawa, T., Honda, Y., Hoshina, T., et al. (2020a). Recurrent shoulder tip pain after ventriculoperitoneal shunt placement associated with infectious peritonitis with Propionibacterium acnes: a case report and review of the literature. J. UOEH 42, 209-216. doi: 10.7888/juoeh.42.209

Suzuki, T., Sutani, T., Nakai, H., Shirahige, K., and Kinoshita, S. (2020b). The microbiome of the meibum and ocular surface in healthy subjects. Investig. Ophthalmol. Vis. Sci. 61:18. doi: 10.1167/iovs. 61.2 .18

Suzuki, Y., Uchida, K., Takemura, T., Sekine, M., Tamura, T., Furukawa, A., et al. (2018). Propionibacterium acnes-derived insoluble immune complexes in sinus macrophages of lymph nodes affected by sarcoidosis. PLoS ONE 13:e0192408. doi: 10.1371/journal.pone.0192408

Tafin, U. F., Corvec, S., Betrisey, B., Zimmerli, W., and Trampuz, A. (2012). Role of rifampin against Propionibacterium acnes biofilm in vitro and in an experimental foreign-body infection model. Antimicrob. Agents Chemother. 56, 1885-1891. doi: 10.1128/AAC.05552-11

Talib, W. H., and Saleh, S. (2015). Propionibacterium acnes augments antitumor, anti-angiogenesis and immunomodulatory effects of melatonin on breast cancer implanted in mice. PLOS ONE 10:124384. doi: 10.1371/journal.pone.0124384

Tamboto, H., Vickery, K., and Deva, A. K. (2010). Subclinical (biofilm) infection causes capsular contracture in a porcine model following augmentation mammaplasty. Plast. Reconstr. Surg. 126, 835-842. doi: 10.1097/PRS.0b013e3181e3b456

Tanabe, T., Ishige, I., Suzuki, Y., Aita, Y., Furukawa, A., Ishige, Y., et al. (2006). Sarcoidosis and NOD1 variation with impaired recognition of intracellular Propionibacterium acnes. Biochim. Biophys. Acta Mol. Basis Dis. 1762, 794-801. doi: 10.1016/j.bbadis.2006.07.006

Tande, A. J., and Patel, R. (2014). Prosthetic joint infection. Clin. Microbiol. Rev. 27, 302-345. doi: 10.1128/CMR.00111-13

Tax, G., Urbán, E., Palotás, Z., Puskás, R., Kónya, Z., Bíró, T., et al. (2016). Propionic acid produced by Propionibacterium acnes strains contributes to their pathogenicity. Acta Derm. Venereol. 96, 43-49. doi: 10.2340/00015555-2154

Thielitz, A., Ansorge, S., Bank, U., Tager, M., Wrenger, S., Gollnick, H., et al. (2008). The ectopeptidases dipeptidyl peptidase IV (DP IV) and aminopeptidase N (APN) and their related enzymes as possible targets in the treatment of skin diseases. Front. Biosci. 13, 2364-2375. doi: 10.2741/2850

Tomida, S., Nguyen, L., Chiu, B. H., Liu, J., Sodergren, E., Weinstock, G. M., et al. (2013). Pan-genome and comparative genome analyses of Propionibacterium acnes reveal its genomic diversity in the healthy and diseased human skin microbiome. mBio 4:13. doi: 10.1128/mBio.00003-13
Trampuz, A., Piper, K. E., Jacobson, M. J., Hanssen, A. D., Unni, K. K., Osmon, D. R., et al. (2007). Sonication of removed hip and knee prostheses for diagnosis of infection. N. Engl. J. Med. 357, 654-663. doi: 10.1056/NEJMoa061588

Triffault-Fillit, C., Ferry, T., Laurent, F., Pradat, P., Dupieux, C., Conrad, A., et al. (2019). Microbiologic epidemiology depending on time to occurrence of prosthetic joint infection: a prospective cohort study. Clin. Microbiol. Infect. 25, 353-358. doi: 10.1016/j.cmi.2018.04.035

Tsuda, K., Yamanaka, K., Linan, W., Miyahara, Y., Akeda, T., Nakanishi, T., et al. (2011). Intratumoral injection of Propionibacterium acnes suppresses malignant melanoma by enhancing Th1 immune responses. PLoS ONE 6:29020. doi: 10.1371/journal.pone.00 29020

Uçkay, I., Dinh, A., Vauthey, L., Asseray, N., Passuti, N., Rottman, M., et al. (2010). Spondylodiscitis due to Propionibacterium acnes: report of twentynine cases and a review of the literature. Clin. Microbiol. Infect. 16, 353-358. doi: 10.1111/j.1469-0691.2009.02801.x

Valanne, S., McDowell, A., Ramage, G., Tunney, M. M., Einarsson, G. G., O’Hagan, S., et al. (2005). CAMP factor homologues in Propionibacterium acnes: a new protein family differentially expressed by types I and II. Microbiology 151, 1369-1379. doi: 10.1099/mic.0.27788-0

Walker, S. P., Tangney, M., and Claesson, M. J. (2020). Sequence-based characterization of intratumoral bacteria-a guide to best practice. Front. Oncol. 10:179. doi: 10.3389/fonc.2020.00179

Wang, Y., Dai, A., Huang, S., Kuo, S., Shu, M., Tapia, C. P., et al. (2014a). Propionic acid and its esterified derivative suppress the growth of methicillinresistant staphylococcus aureus USA300. Beneficial Microbes 5, 161-168. doi: 10.3920/BM2013.0031

Wang, Y., Kuo, S., Shu, M., Yu, J., Huang, S., Dai, A., et al. (2014b). Staphylococcus epidermidis in the human skin microbiome mediates fermentation to inhibit the growth of Propionibacterium acnes: implications of probiotics in acne vulgaris. Appl. Microbiol. Biotechnol. 98, 411-424. doi: 10.1007/s00253-013-5394-8

Wang, Y., Telesford, K. M., Ochoa-Repáraz, J., Haque-Begum, S., Christy, M., Kasper, E. J., et al. (2014c). An intestinal commensal symbiosis factor controls neuroinflammation via TLR2-mediated CD39 signalling. Nat. Commun. 5:5432. doi: 10.1038/ncomms5432

Werner, J. L., Escolero, S. G., Hewlett, J. T., Mak, T. N., Williams, B. P., Eishi, Y., et al. (2017). Induction of pulmonary granuloma formation by Propionibacterium acnes is regulated by MyD88 and Nox2. Am. J. Respir. Cell Mol. Biol. 56, 121-130. doi: 10.1165/rcmb.20160035OC

Wollein Waldetoft, K., Råberg, L., and Lood, R. (2020). Proliferation and benevolence-a framework for dissecting the mechanisms of microbial virulence and health promotion. Evol. Appl. 13, 879-888. doi: 10.1111/eva.12952

Wollenberg, M. S., Claesen, J., Escapa, I. F., Aldridge, K. L., Fischbach, M. A., and Lemon, K. P. (2014). Propionibacterium-produced coproporphyrin III induces staphylococcus aureus aggregation and Biofilm formation. mBio 5, 1-10. doi: 10.1128/mBio.01286-14

Xia, X., Li, Z., Liu, K., Wu, Y., Jiang, D., and Lai, Y. (2016). Staphylococcal LTA-induced miR-143 inhibits Propionibacterium acnes-mediated inflammatory response in skin. J. Investig. Dermatol. 136, 621-630. doi: $10.1016 /$ j.jid.2015.12.024

Yamaguchi, T., Costabel, U., McDowell, A., Guzman, J., Uchida, K., Ohashi, K., et al. (2021). Immunohistochemical detection of potential microbial antigens in granulomas in the diagnosis of sarcoidosis. J. Clin. Med. 10:983. doi: $10.3390 / \mathrm{jcm} 10050983$

Yang, F., Ma, Q., Lei, L., Huang, J., Ji, Q., Zhai, R., et al. (2014). Specific humoral immune response induced by Propionibacterium acnes can prevent Actinobacillus pleuropneumoniae infection in mice. Clin. Vaccine Immunol. 21, 407-416. doi: 10.1128/CVI.00667-13

Yang, T. S., and Liu, T. T. (2004). Optimization of production of conjugated linoleic acid from soybean oil. J. Agric. Food Chem. 52, 5079-5084. doi: 10.1021/jf0401017

Yu, Y., Champer, J., Agak, G. W., Kao, S., Modlin, R. L., and Kim, J. (2016). Different Propionibacterium acnes phylotypes induce distinct immune responses and express unique surface and secreted proteomes. J. Investig. Dermatol. 136, 2221-2228. doi: 10.1016/j.jid.2016.06.615 
Zeller, V., Ghorbani, A., Strady, C., Leonard, P., Mamoudy, P., and Desplaces, N. (2007). Propionibacterium acnes: an agent of prosthetic joint infection and colonization. J. Infection 55, 119-124. doi: 10.1016/j.jinf.2007.02.006

Zeng, R., Xu, H., Liu, Y., Du, L., Duan, Z., Tong, J., et al. (2019). miR146a inhibits biofilm-derived Cutibacterium acnes-induced inflammatory reactions in human keratinocytes. J. Invest. Dermatol. 139, 2488-2496. doi: 10.1016/j.jid.2019.03.1161

Zhu, W., Winter, M. G., Byndloss, M. X., Spiga, L., Duerkop, B. A., Hughes, E. R., et al. (2018). Precision editing of the gut microbiota ameliorates colitis. Nature 553, 208-211. doi: 10.1038/nature25172

Zouboulis, C. C., Jourdan, E., and Picardo, M. (2014). Acne is an inflammatory disease and alterations of sebum composition initiate acne lesions. J. Eur. Acad. Dermatol. Venereol. 28, 527-532. doi: 10.1111/jdv. 12298
Conflict of Interest: HB, HG, and RL are members of the scientific advisory board of S-Biomedic. This had no influence on this work.

The remaining author declares that the research was conducted in the absence of any commercial or financial relationships that could be construed as a potential conflict of interest.

Copyright (c) 2021 Brüggemann, Salar-Vidal, Gollnick and Lood. This is an openaccess article distributed under the terms of the Creative Commons Attribution License (CC BY). The use, distribution or reproduction in other forums is permitted, provided the original author(s) and the copyright owner(s) are credited and that the original publication in this journal is cited, in accordance with accepted academic practice. No use, distribution or reproduction is permitted which does not comply with these terms. 